\title{
2 Measuring Cognitive Effort Without Difficulty
}

3

4

5

6

7

Hugo Fleming ${ }^{\mathrm{a}, \mathrm{b}^{*}}$, Oliver J. Robinson ${ }^{\mathrm{a}} \&$ Jonathan Roiser ${ }^{\mathrm{a}}$

8

9

10 a Institute of Cognitive Neuroscience, University College London, 17 Queen Square, London,

$11 W C 1 N 3 A Z, U K$

$12{ }^{\mathrm{b}}$ MRC Cognition and Brain Sciences Unit, 15 Chaucer Road, Cambridge, CB2 7EF, UK

13

14

$15 *$ Corresponding author: hugo.fleming@mrc-cbu.cam.ac.uk

16

17

18

19 


\section{Abstract}

2 An important finding in the cognitive effort literature has been that sensitivity to the costs of

3 effort varies between individuals, suggesting that some people find effort more aversive than

4 others. It has been suggested this may explain individual differences in other aspects of

5 cognition; in particular that greater effort sensitivity may underlie some of the symptoms of

6 conditions such as depression and schizophrenia. In this paper we highlight a major problem

7 with existing measures of cognitive effort that hampers this line of research, specifically the

8 confounding of effort and difficulty. This means that behaviour thought to reveal effort costs

9 could equally be explained by cognitive capacity, which influences the frequency of success

10 and thereby the chance of obtaining reward. To address this shortcoming we introduce a new

11 test, the Number Switching Task (NST), specially designed such that difficulty will be

12 unaffected by the effort manipulation and can easily be standardised across participants. In a

13 large, online sample we show that these criteria are met successfully and reproduce classic

14 effort discounting results with the NST. We also demonstrate the use of Bayesian modelling

15 with this task, producing behavioural parameters which can then be associated with other

16 measures, and report a preliminary association with the Need for Cognition scale.

19 Keywords: anhedonia, cognitive effort, computational psychiatry, depression, individual

20 differences, new measures 


\section{Introduction}

2 Cognitive effort, our ability to vary the depth of our engagement with a cognitive task,

3 influences a raft of fundamental cognitive processes including attention (Kahneman, 1973),

4 working memory (Westbrook et al., 2013), cognitive control (Braver, 2012; Shenhav,

$5 \quad$ Botvinick \& Cohen, 2013) and decision-making (Ortega et al., 2015; Toplak, West \&

6 Stanovich, 2011). Consequently, there is substantial interest in both measuring cognitive

7 effort and understanding the factors that determine when and how much effort is exerted in

8 different situations. Unfortunately, cognitive effort is challenging to study for the very same

9 reason - because it is so entangled with other processes, there is considerable potential for

10 confounding, and attempts to measure cognitive effort must therefore be careful to isolate

11 effort from other factors that may influence performance.

13 One particular problem - the conflation of effort and difficulty — has not to our knowledge been addressed. Current methods for studying cognitive effort involve assessing participants' preferences for different cognitive tasks: avoidance of more demanding tasks is interpreted as evidence of underlying effort costs, and these are quantified by examining how participants trade off the demand against rewards (a phenomenon termed effort discounting; see Westbrook \& Braver, 2015). However, more demanding tasks may also have lower rates of success, and therefore of obtaining reward, giving rise to another form of discounting (this time by the probability of reward) that would cause avoidance of the more demanding tasks 21 in exactly the same way.

23 Consider for example the N-back working memory task, which is frequently used in studies of cognitive effort (see e.g. Westbrook et al., 2013). Higher levels of the N-back feel more effortful, but they are also intrinsically more difficult to perform accurately, because with 
1 more items to hold in memory, the maximum precision with which each item can be

2 maintained is lower (Bays, Catalao \& Husain, 2009). If we observe discounting of the value

3 of the task as the N-back level increases, it is impossible to say to what extent this is due to

4 the greater effort required or the lower probability of completing a trial successfully and

5 gaining reward. Similar arguments can be made for other effort manipulations, including

6 visual attention (e.g. Apps, 2015), auditory attention (e.g. McLaughlin et al., 2021; Crawford,

7 Eisenstein et al., 2022) and response conflict tasks (e.g. McGuire \& Botvinick, 2010;

8 Schmidt et al., 2012). This confounding may cause overestimation of participants' cognitive

9 effort sensitivity (because both effort and probability discounting are contributing to the

10 observed behaviour). In order to dissociate these processes, it is essential that measures of

11 cognitive effort hold difficulty constant when manipulating task demand.

13 It is important too that the difficulty of the task can be standardised across participants, as is 14 usual in tasks manipulating physical effort (Chong et al., 2016; Husain \& Roiser, 2018). For

15 example, in physical effort tasks measuring grip strength (e.g. Bonnelle et al., 2016), the

16 different levels are typically normalised relative to participants' maximum grip force, so that

17 while the absolute difficulty of the task (the grip force required) may differ between

18 participants, the relative difficulty (the proportion of maximum capacity required) is held

19 constant; crucially, therefore, participants are all able to achieve comparable levels of

20 performance. Likewise, in cognitive effort tasks, we need to take account of baseline

21 differences in cognitive capacity (for example, differences in working memory capacity or processing speed). In tasks that do not standardise the difficulty, the problem of confounding again rears its head - if two participants apparently differ in the subjective value they ascribe to a task, we cannot say to what extent this reflects genuine differences in sensitivity to effort, as opposed simply to differences in the probability of achieving success. This is a particular 
1 concern with regards to psychiatric research into conditions such as depression and

2 schizophrenia, where cognitive impairment is a core symptom (Mesholam-Gately et al.,

3 2009; Rock et al., 2014) and therefore patients are likely to find the task fundamentally more

4 difficult than healthy controls.

5

6 Some attempts have been made previously to take account of this issue. For example,

7 Westbrook et al. (2013) statistically control for the effect of performance on subjective value

8 in a regression model. However, this involves conditioning on a variable (performance)

9 which is causally posterior to the outcome variable of the regression (subjective value). It is

10 better to standardise the task itself, but to our knowledge this has been attempted only once

11 before, by Ang et al. (2022), who devised a task they called the Cognitive Effort Motivation

12 Task (CEMT). Ang et al. probed effort by asking participants to remember the location of

13 one or more squares on a checkerboard, and normalised the difficulty for each participant by

14 reducing the total number of squares on the grid until they could achieve at least $80 \%$

15 accuracy during a training phase. However, although the CEMT has resolved the issue of

16 confounding between participants, it still suffers from confounding within participants - as

17 with the N-back task discussed above, higher demand levels in the CEMT give participants

18 more information to hold in memory, such that both effort and the intrinsic difficulty of the

19 task covary.

\section{A new cognitive effort measure - the Number Switching Task}

22 The purpose of the present study was therefore to develop a task that distinguishes cognitive

23 effort discounting from probability discounting completely. Specifically, we targeted two

24 main criteria: the manipulation of effort demand should not affect the probability of success;

25 and it should be possible to standardise the task difficulty by reference to each participant's 
1 baseline ability. While other tasks have been able to satisfy one or other of these criteria, the

2 key significance of our task is that it meets both of them. Two further considerations were

3 that the task should have several levels of effort demand, so that we could examine

4 parametric responses to the manipulation across a reasonable dynamic range, and also that it

5 should be optimised for use online, where it is possible to obtain much larger sample sizes

6 more practically than through in-person testing.

7

8 We developed the Number Switching Task (NST), which involves categorising each digit in

9 a nine-digit sequence as either even or odd. By changing the frequency of switching between

10 odd and even we can manipulate the effort required but, crucially, it is only the order of the

11 digits that changes - the actual content of the trials is all the same. This means that regardless

12 of effort level, participants are completing the same set of operations on every trial, so the

13 difficulty of the task - the information processing demands - is held constant (this

14 distinguishes the NST from working memory based effort tasks, in which higher effort levels

15 contain more information content to remember). Significantly, in the NST we can also

16 standardise the difficulty of the task, by calibrating the time participants are allowed to

17 complete each sequence, such that all participants are capable of achieving comparable levels

18 of success.

20 Note that while this task is superficially similar to the Demand Selection Task (Kool et al.,

21 2010), which also involves categorising digits, the manipulation of effort is rather different:

22 the Demand Selection Task employs a Stroop-like structure of switching between different

23 response rules, whereas in the NST it is the switching between odd and even itself that is

24 effortful. This means that while the Demand Selection Task only compares two conditions,

25 high vs. low switching, the NST is capable of measuring a wider range of effort levels (here 
1 we use 4 effort levels). In addition the NST allows us to examine the interacting effect of

2 reward on participants' choices, unlike the Demand Selection Task which probes effort alone.

3

4 The primary aim of this study was to validate the NST by testing the prediction that the effort

5 manipulation will elicit the classic effort discounting effect without affecting the difficulty as

6 measured by the rate of success. We also present some secondary analyses including

7 Bayesian modelling and an assessment of preliminary associations with cognitive traits

8 relevant to depression and anhedonia. 


\section{Method}

\section{Preregistration}

3 This study was preregistered on the Open Science Framework

4 (doi:10.17605/OSF.IO/8Y7P9). There were no deviations from this plan.

6 Participants

7 Participants were recruited through the online platform Prolific (www.prolific.co/). To be

8 eligible, they had to be aged 18-60, with no history of a diagnosed mental health condition,

9 and could not have taken part in any of the pilot experiments for this study. They also had to

10 use a computer - smartphones or tablets were not allowed.

12 In our preregistration we calculated a minimum required sample size of 259 participants in

13 order to detect an effect of $r=.2$ with $90 \%$ power and alpha $=.05$ (two-tailed). We recruited

14 more than this, to allow for withdrawals and exclusions, so that the sample included 306

15 participants who completed the experiment. Of these, three were excluded because they

16 refreshed the web page part way through; nine were excluded because they repeatedly failed

17 the familiarisation phase of the effort task; and four were excluded because they failed

18 attention checks in the questionnaires. This left 290 participants with data included in the

19 final analysis. The age and education distribution of this sample is provided in Table 1. 


\begin{tabular}{|c|c|}
\hline \multicolumn{2}{|l|}{ Table 1. The age and education distribution of participants. } \\
\hline Age group & N \\
\hline $18-21$ & 69 \\
\hline $22-25$ & 61 \\
\hline $25-30$ & 70 \\
\hline $30-35$ & 37 \\
\hline $35-40$ & 20 \\
\hline $40-45$ & 13 \\
\hline $45-50$ & 7 \\
\hline $50-55$ & 9 \\
\hline $55-60$ & 4 \\
\hline & $\mathbf{N}$ \\
\hline Education & 96 \\
\hline Secondary Education/High School & 16 \\
\hline Higher Apprenticeship & 7 \\
\hline Foundation Degree & 106 \\
\hline Bachelor's Degree/Degree Apprenticeship & 58 \\
\hline Master's Degree & 7 \\
\hline PhD & \\
\hline
\end{tabular}

3 Procedure

4 From Prolific, participants were automatically directed to another website, Gorilla

5 (www.gorilla.sc/), where the study was hosted. There they completed the Cognitive Effort

6 Task, followed by eight questionnaires. At the end of the study, they were redirected back to

7 Prolific via a unique URL, which allowed them to prove they had completed all the tasks; if

8 instead they returned to Prolific manually (without this URL), their data was flagged and we

9 checked whether they had actually completed all the tasks or not. On average, the entire study

10 took approximately 45 minutes, from signing up to returning to Prolific, and participants 
1 were paid a flat rate of $£ 5$ plus a performance bonus of 1 pence per 3 points won on the effort task (on average participants won around $£ 1.50$ in bonuses).

\section{The Number Switching Task}

5 The structure of the task is shown in Figure 1. On each trial, participants were offered a

6 reward (3, 6, 9 or 12 points, corresponding to $1,2,3$ or $4 \mathrm{p}$ of real money, respectively) to

7 complete an effortful task with a specified level of demand. If they accepted this challenge

8 they had to complete the task successfully to win the reward; if they rejected it, they avoided

9 performing the task, but won no points and, after a timeout of $2500 \mathrm{~ms}$, proceeded to the next

10 offer.

12 The effortful task itself was to categorise each of the digits in a random sequence of the

13 numbers one to nine as either odd or even. The effort of this task scales with the frequency of

14 switching between odd and even digits, allowing us to define four levels of demand: the

15 lowest level, referred to in the task as $20 \%$, contained either 1 or 2 switches; the next level

$16(40 \%) 3$ or 4 switches; the $60 \%$ level 5 or 6 switches; and the highest level, $80 \%$, had 7 or 8

17 switches. On any given trial, the precise number of switches was determined at random to

18 prevent the sequences becoming predictable. Participants were not explicitly told about the

19 relationship between effort level and number of switches but instead learned this over the

20 course of the familiarisation and main phases of the experiment. 


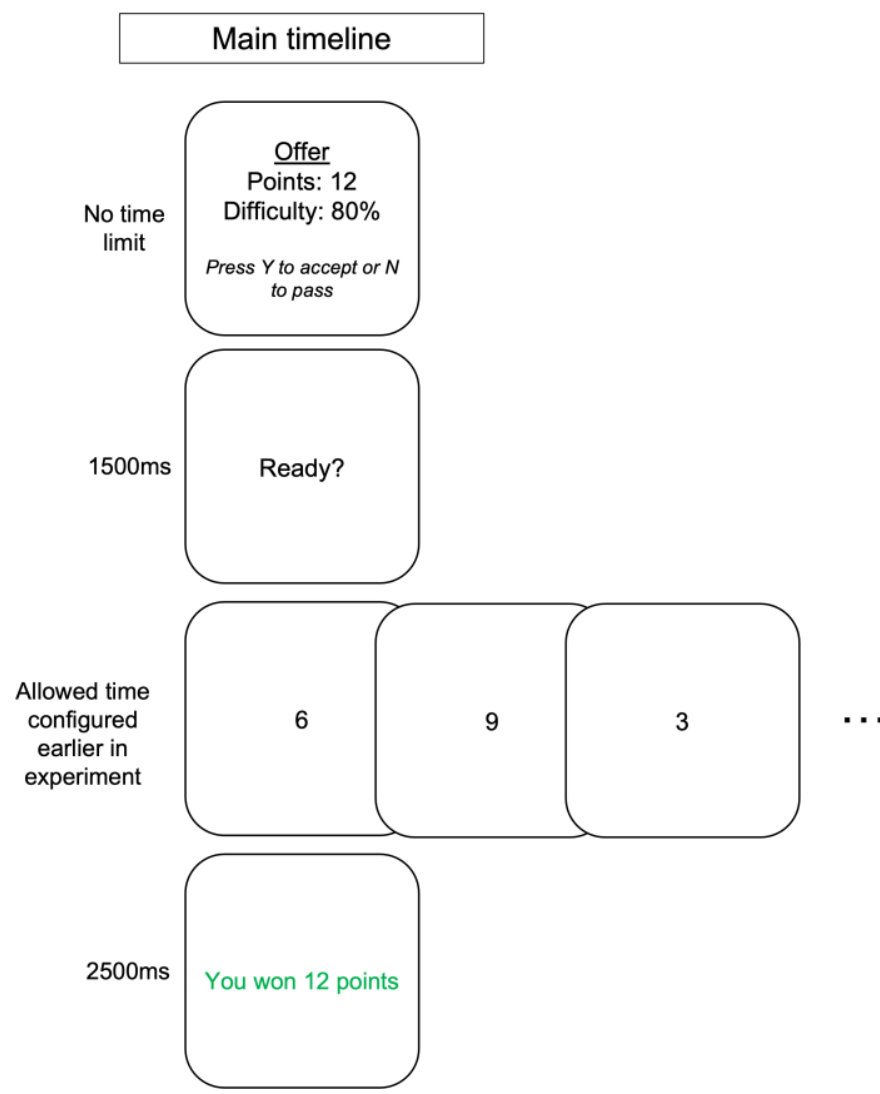

Alternative outcomes

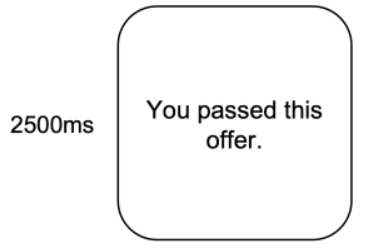

Figure 1. Number Switching Task trial structure. Participants chose whether or not to perform an effortful task depending on the points and effort level offered. If they accepted the offer, they were shown a random sequence of the digits $1-9$, and had to indicate (by pressing the ' $\mathrm{f}$ ' or ' $\mathrm{j}$ ' keys) whether each of the digits was even or odd. Sequences with more frequent switching between odd and even were more effortful. To win the points on offer, participants had to categorise at least 8 of the 9 digits correctly and complete the sequence within the allowed time (which was calibrated to each individual). In the above figure, the 'alternative outcomes' show screens that participants saw if they passed an offer, or if they failed the trial owing to too many errors or timing out.

3 As each digit appeared on the screen in sequence, participants responded, as soon as they saw

4 the digits, using the ' $\mathrm{f}$ ' and ' $\mathrm{j}$ ' keys to indicate whether it was 'odd' or 'even' (key mapping

5 was counterbalanced across participants). Instructions about these responses were provided

6 on an instruction screen at the start of the task. While the individual categorisations were self-

7 paced, meaning the next digit did not appear on the screen until a response had been made to

8 the current item, there was a time limit for completing the overall sequence. A trial was 
1 marked as 'correct' only if the sequence was finished within this limit and with no more than

2 one wrong response. 'Incorrect' sequences were not rewarded.

3

4 Importantly, this allowed us to standardise the difficulty across participants: by calibrating

5 the allowed time based on performance during an earlier familiarisation phase, we ensured

6 that all participants had similar success rates on the task.

7

$8 \quad$ Phases of the task

9 Prior to embarking on the full task, participants progressed through instructions and practice

10 rounds, followed by a longer familiarisation phase. This latter phase was used to calibrate the

11 time limit for the sequences in the main phase of the task. See Figure 2. 


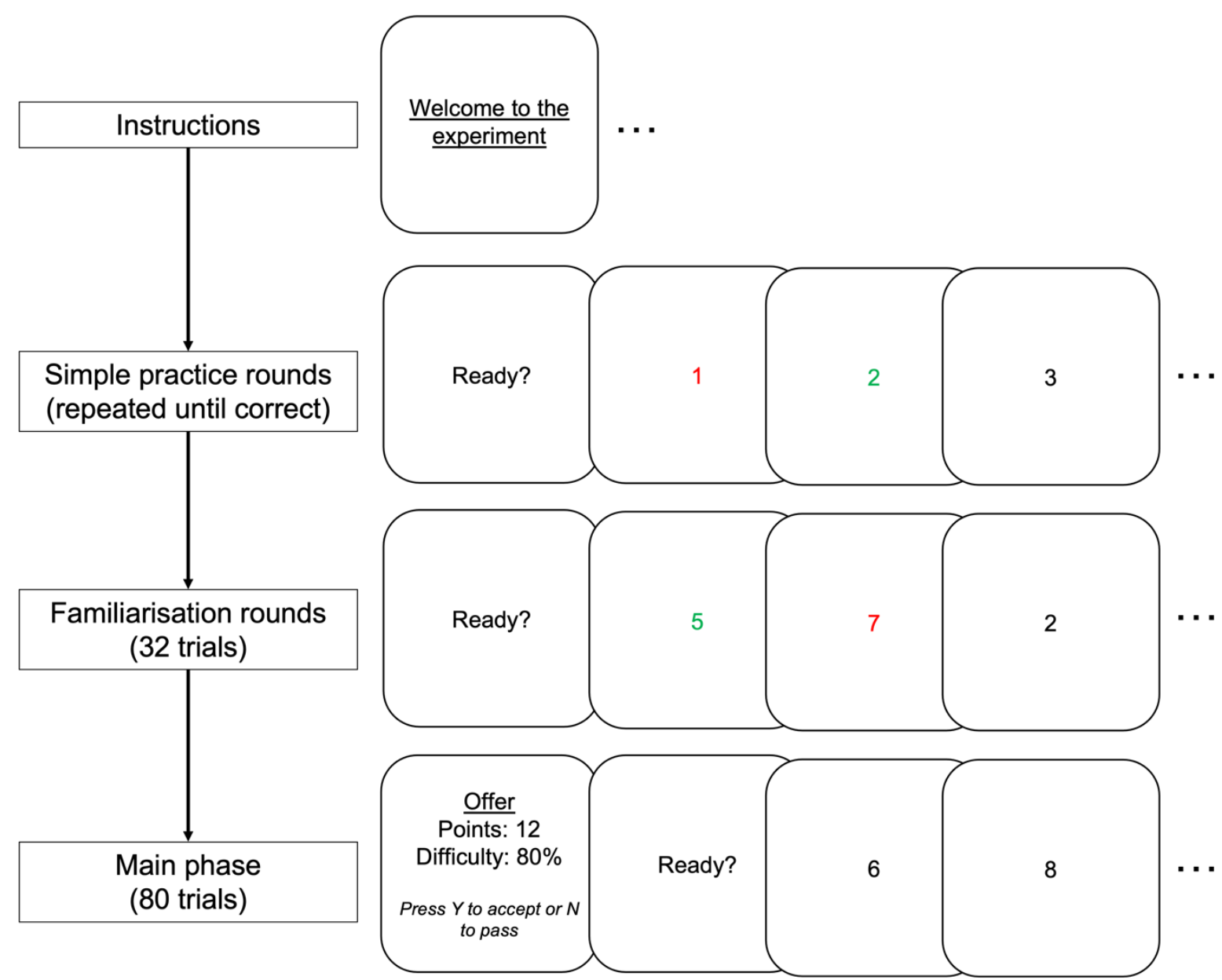

Figure 2. Overall structure of the different phases of the task. See main text for detailed description of each phase.

2

3 The instruction phase informed participants which keys they should press to categorise each

4 digit as even or odd. Then, in an initial practice round, they were shown a sequence of the

5 digits one to nine in ascending order, and they had to categorise all of these correctly to

6 continue; if they made a single mistake, this round was repeated. We thus ensured that

7 participants' keys were working and that they understood the basic premise of the task.

8 Unlike in the later, main phase of the task, here there was no time limit for completing the

9 sequences. In addition, after participants gave their response the digits changed to green or

10 red to indicate whether the response was correct or not (unlike in the main phase of the task

11 where participants only received feedback at the end of the sequence). 
1 The subsequent familiarisation phase was similar-participants completed a set of 32

2 sequences, again without the offer framing or the time limit — but now the digits were

3 presented in a randomised order. This phase comprised four trials of each of the eight

4 possible numbers of switches. Although there was no time limit, participants were now

5 instructed to respond as quickly as possible while still trying to complete each sequence

6 correctly.

7

8 To progress through the familiarisation phase to the main task, participants had to achieve at

9 least $50 \%$ correct responses on the most difficult 8-switch trials; if they failed more than $50 \%$

10 of these trials they were given one opportunity to repeat this stage; if they failed again they

11 were excluded.

12

13 For participants who passed the familiarisation phase, we calculated their time allowed for

14 the main phase sequences as the median time to complete the hardest, 8-switch trials plus

15 500ms. During piloting we observed that this provided a good balance between providing

16 sufficient time pressure to elicit the effort effects while ensuring that the task was possible

17 within the maximum completion time for all participants.

19 Finally, participants completed the main phase of the task, in which the full trial structure was

20 used (as depicted in Figure 1). After 10 example trials in which participants were introduced

21 to and gained experience with the offer framing, the main phase itself comprised a total of 80

22 trials - five trials of each of the 16 possible offer combinations, in a random order. 


\section{Questionnaire measures}

2 Following the NST, participants completed a number of questionnaires. In all cases,

3 participants gave their responses by moving a horizontal slider (which defaulted to the

4 centre).

5

6 First was the NASA Task Load Index (Hart \& Staveland, 1988), henceforth the 'Subjective

7 Task Load', which asked participants to give their subjective rating (over six subscales) of

8 the level of demand felt while performing each of the effort levels in the task. Subsequently

9 there were seven questionnaires assessing different traits: the Cognitive Complaints

10 Inventory (Iverson \& Lam, 2013), the Fatigue Severity Scale (Krupp, LaRocca, Muir-Nash \&

11 Steinberg, 1989), the International Physical Activity Questionnaire Short Form (Lee,

12 Macfarlane, Lam \& Stewart, 2011), the Need for Cognition six-item scale (Coelho, Hanel \&

13 Wolf, 2018), the Temporal Experience of Pleasure Scale (Gard, et al. 2006) and the Zung

14 Depression Scale (Zung, 1965). Further detail on these questionnaires and their scoring is

15 provided in the Supplement.

16

17 We appended catch questions (e.g. "Select 'very much' for this question") to two

18 questionnaires, the Cognitive Complaints Inventory and the Need for Cognition Scale, to

19 identify participants who were not paying attention. We placed these at the end of the

20 questionnaires to avoid interfering with the psychometric properties of the measures

21 themselves. 


\section{Preregistered analyses}

3 The main dependent variable on the NST was the proportion of offers accepted for each

4 combination of reward and effort level. We also recorded participants' accuracy and completion times for the odd/even categorisation task - these were of course conditional on

6 participants accepting the offer in the first place and, in the case of the completion times,

7 completing the sequence within the time allowed and with no more than one mistake allowed.

9 Our primary analysis was a multilevel (mixed effects) ANOVA. This was used because 10 multilevel ANOVAs can accommodate unbalanced designs, which arise in this task because

11 participants could choose to accept or reject trials at will, resulting in secondary measures

12 (success rate and completion time) with different numbers of trials from each participant.

13 These ANOVAs contained fixed effects of reward and effort and their interaction, and 14 varying intercepts across subjects.

16 For analysis of the Subjective Task Load questionnaire, six multilevel ANOVAs were

17 constructed, one for each of the constituent scales of the index, using a fixed effect of effort 18 level and varying intercepts across subjects.

20 Throughout these analyses, we further investigated any significant effects indicated by the 21 ANOVAs using post-hoc simple effects ANOVAs and paired-samples $t$-tests as appropriate.

22 Note that, unlike the multilevel ANOVAs, the $t$-tests require complete cases. This results in 23 differing degrees of freedom across analyses, as some participants had to be excluded from specific post-hoc analyses of success rates or completion times if they had not completed any trials at a particular reward or effort level. 


\section{Exploratory analyses}

2 Bayesian Modelling

3 We considered twelve models, all variations on a logistic regression, listed in Table 2. The

4 characteristic mathematical form of these models is provided in Equation 1. This particular

5 set of equations represents Model 9 - all of the other models can be constructed by modifying

6 one or more components of this model.

7

8 Equation 1: 
Table 2. Specification of models fitted to the Number Switching Task. We fitted and compared twelve models in total, containing different terms. $\mathrm{I}=$ intercept, $\mathrm{R}=$ reward, $\mathrm{E}=$ effort. The prefix $\mathrm{f}$ or $\mathrm{v}$ indicates whether this term was fixed (one parameter estimated for the whole population) or varying (individual effects estimated for each participant, constrained by a hierarchical prior).

\begin{tabular}{|l|l|}
\hline $\begin{array}{c}\text { Model } \\
1\end{array}$ & $\mathrm{fI}$ \\
\hline 2 & $\mathrm{fI}+\mathrm{fR}+\mathrm{fE}$ \\
\hline 3 & $\mathrm{vI}+\mathrm{fR}+\mathrm{fE}$ \\
\hline 4 & $\mathrm{vI}+\mathrm{vR}+\mathrm{vE}$ \\
\hline 5 & $\mathrm{fI}+\mathrm{fR}+\mathrm{fE}+\mathrm{f} E^{2}$ \\
\hline 6 & $\mathrm{vI}+\mathrm{fR}+\mathrm{fE}+\mathrm{f} E^{2}$ \\
\hline 7 & $\mathrm{vI}+\mathrm{vR}+\mathrm{vE}+\mathrm{v} E^{2}$ \\
\hline 8 & $\mathrm{vI}+\mathrm{vR}+\mathrm{vE}{ }^{2}$ \\
\hline 9 & $\mathrm{vI}+\mathrm{vR}+\mathrm{v} R^{2}+\mathrm{vE}$ \\
\hline 10 & $\mathrm{vI}+\mathrm{v} R^{2}+\mathrm{vE}$ \\
\hline 11 & $\mathrm{vI}+\mathrm{vR}+\mathrm{v} R^{2}+\mathrm{vE}+\mathrm{v} E^{2}$ \\
\hline 12 & $\mathrm{vI}+\mathrm{v} R^{2}+\mathrm{v} E^{2}$ \\
\hline
\end{tabular}

3 The subject-level parameters were all given hierarchical priors which were determined

4 through a process of prior predictive checking. Details are given in the Supplement.

6 We standardised the values of the predictors (the reward and effort levels), for computational

7 and arithmetical simplicity. Note that this affects the interpretation of absolute parameter

8 values from the model.

10 The models were fitted using Markov Chain Monte Carlo simulation in Stan (Stan

11 Development Team, 2021). Sampling was run for four chains each with 1000 iterations.

12 Subsequent to fitting, we carried out the recommended standard diagnostics (visual

13 inspection of the chains, no divergences or treedepth warnings, E-BFMI $<0.3$, effective

14 sample size $>400$, split- $\hat{R}<1.01$; Betancourt, 2018) and found no issues. We also inspected 
1 the posterior predictions for the winning model and observed a good overall fit to the data 2 (see Figure S7).

3

$4 \quad$ Structural Equation Modelling

5 We used confirmatory factor analysis (CFA) to fit several potential factor structures to the

6 questionnaire data. We identified the best-fitting structure and inserted this into a structural

7 equation model (SEM), with which we sought to predict the behavioural parameters

8 estimated for each subject (intercept, reward and effort sensitivity) from their cognitive trait

9 scores.

10

11 Computing environment and packages

12 Analyses were conducted in $\mathrm{R}$ version 3.5.3 ( $\mathrm{R}$ Core Team, 2019). We used the R package

13 'Ime4' (1.1-21; Bates et al., 2015) to fit the multilevel ANOVAs and 'rstatix' (0.6.0;

14 Kassambara, 2020) to conduct the post-hoc tests. Bayesian models were fitted in Stan using

15 CmdStanR (0.3.0, Gabry, \& Češnovar). SEM was conducted in Lavaan (Rosseel, 2012).

16

17 


\section{Results}

2 Preregistered Analyses

\section{Number Switching Task}

$4 \quad$ Proportion of Offers Accepted

5 The proportions of offers accepted at each level of reward and effort are plotted in Figure 3.

6 These show a significant reward-by-effort interaction, $F(1,4347)=30.8, p<.001$,

$7 \quad \eta^{2}$ partial $=.04$, consistent with participants treating the effort level as an economic cost.

8 Specifically, the value of a reward was progressively discounted as the effort required to

9 obtain it increased, but this discounting was shallower when the reward offered is greater.

10 Despite this flattening as reward increased, the effort effect was still significant at every

11 reward level in post-hoc ANOVAs (all $p s<.001$ ). The main effects of reward and effort were

12 also both significant, $F(1,4347)=108, p<.001$, and $F(1,4347)=84.4, p<.001$ respectively.

13 Full descriptive statistics are provided in Table S1.

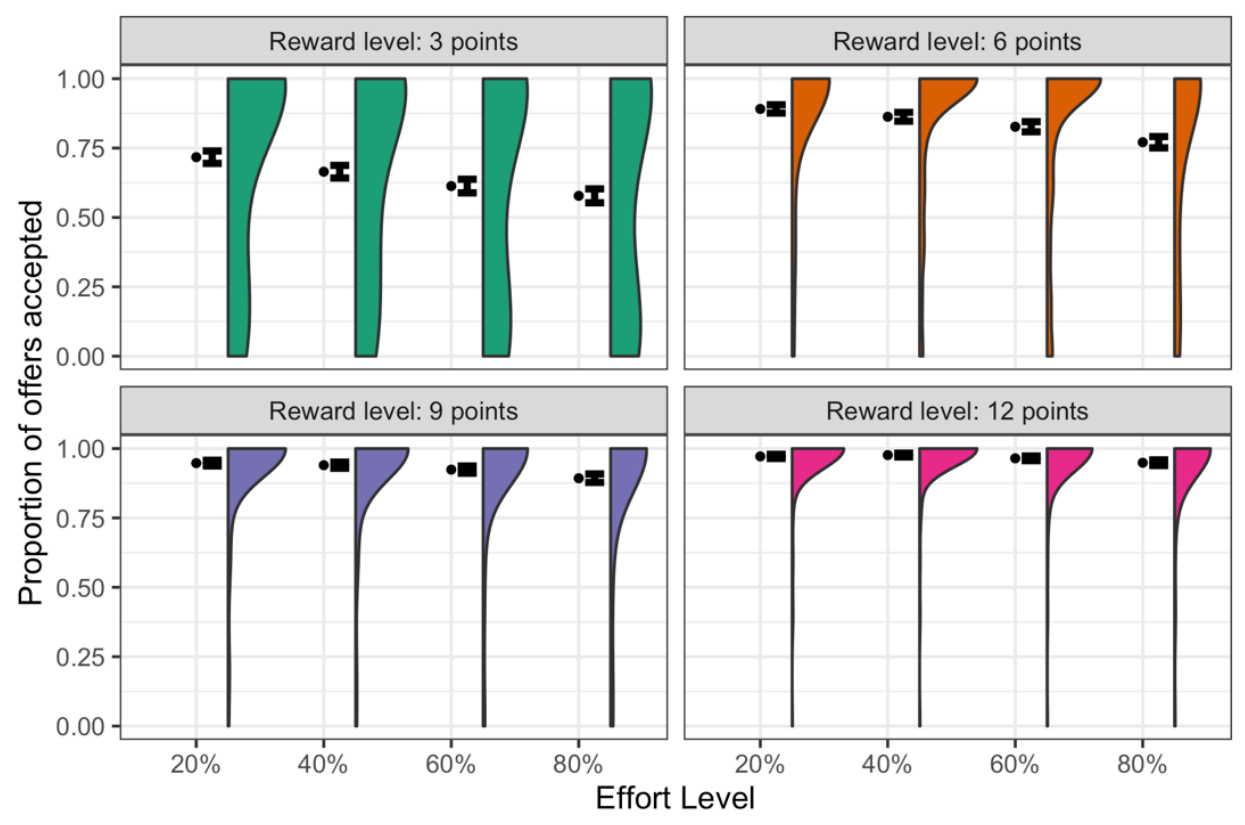

Figure 3. Number Switching Task: proportion of offers accepted. Figure shows the mean, standard error and distribution of the proportion of offers accepted for each combination of reward and effort level. See also Figure S9 for the same plot without faceting. 
1 Success Rate

2 The success rate for each level of reward and effort is plotted in Figure 4. The only

3 statistically significant effect was that of reward, $F(1,4024)=68.1, p<.0001, \eta^{2}$ partial $=0.08$,

4 with participants more likely to complete the sequence correctly as the offered reward

5 increased (Table 3), consistent with higher rewards being more motivating. Post-hoc $t$-tests

6 indicated that this was driven primarily by the increase in success rates between the 3 and 6

7 point reward levels, $t(272)=3.01, p=.008, d=0.18$, while the differences between 6 and 9

8 points, and 9 and 12 points did not achieve significance after Bonferonni-adjusting for

9 multiple comparisons ( $p s=.10$ and .07 , and, $d s=0.13$ and 0.14 , respectively). Full

10 descriptive statistics are provided in Table S2.

12 The effort level had no significant effect on the success rate, $F(1,4024)=2.18, p=.14$, and

13 the reward-by-effort interaction was also non-significant, $F(1,4024)=0.380, p=.54$.

15 Importantly, the success rate varied relatively little across participants (overall mean $=0.90$,

$16 \mathrm{SD}=0.11$ ), suggesting the standardisation of difficulty had been successful.

17

18

19

20

21

22 
Table 3. Number Switching Task: Descriptive statistics for the proportion of trials completed successfully (across reward levels).

\section{$\mathbf{P}($ Success $)$}

\begin{tabular}{|c|c|c|}
\hline Reward (points) & N & Mean (SD) \\
\hline 3 & 273 & $0.86(0.18)$ \\
\hline 6 & 273 & $0.89(0.12)$ \\
\hline 9 & 273 & $0.91(0.10)$ \\
\hline 12 & 273 & $0.92(0.10)$ \\
\hline
\end{tabular}

Note. To be marked as correct, sequences had to be completed within the time limit and with no more than one error. These data only include complete cases, i.e. where participants attempted at least one trial for each level of reward. $\mathrm{SD}=$ standard deviation.
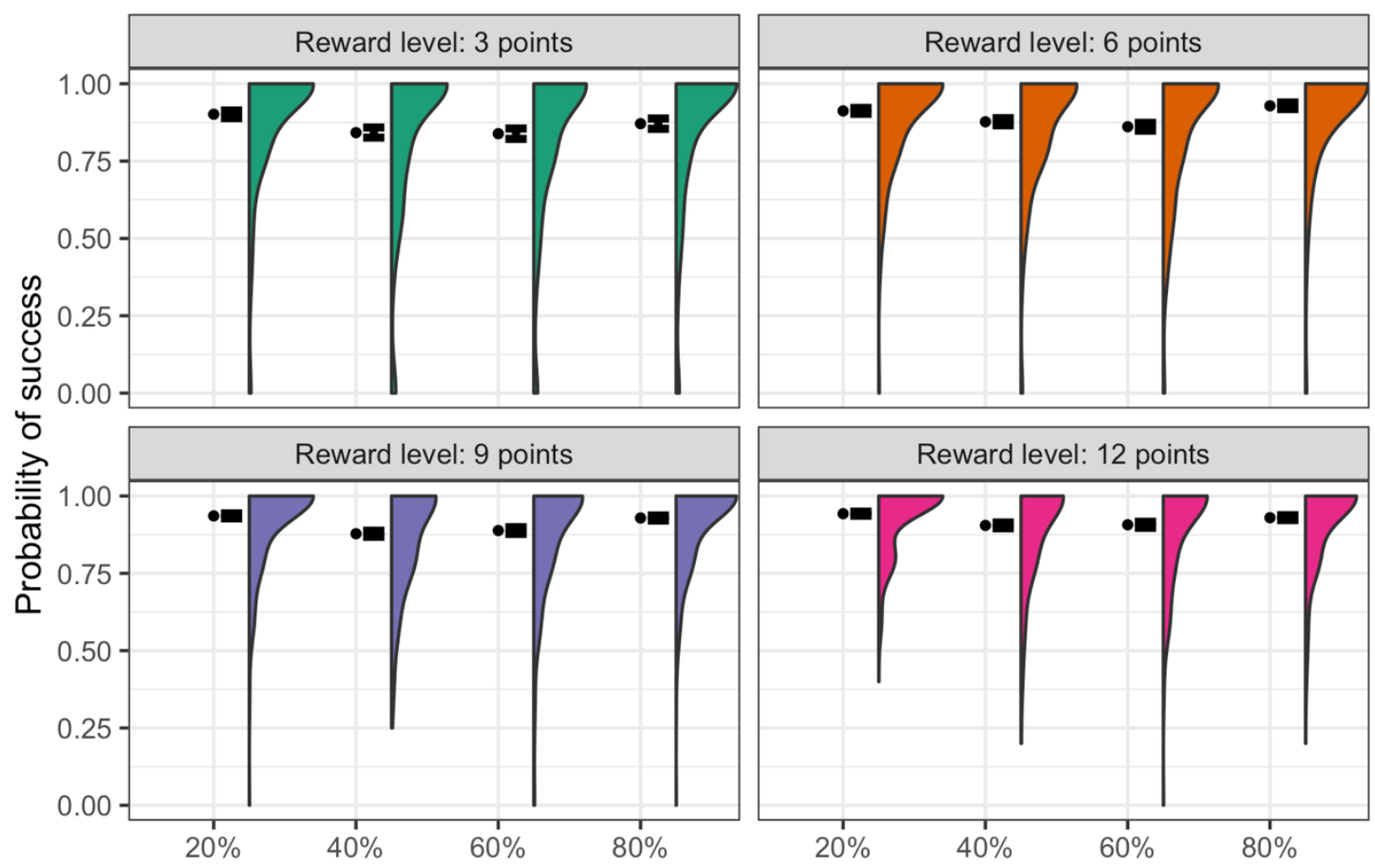

Effort Level

Figure 4. Number Switching Task: proportion of trials completed successfully. Figure shows the mean, standard error and distribution for each combination of reward and effort level. Trials were marked as 'correct' if they were completed within the allowed time, with no more than one error. See also Figure S9 for the same plot without faceting. 


\section{$1 \quad$ Completion Times}

2 Completion times, expressed as a proportion of each participant's maximum allowed time,

3 are plotted in Figure 5. Full descriptive statistics are provided in Table S3. The overall mean

4 (SD) maximum allowed time was $8953 \mathrm{~ms}(1700 \mathrm{~ms})$. There were significant main effects of

5 both reward, $F(1,4014)=10.1, p=.002, \eta^{2}$ partial $=.03$, and effort, $F(1,4014)=610, p<.001$,

$6 \eta_{\text {partial }}^{2}=.52$. The interaction effect was non-significant, $F(1,4014)=0.56, p=.45 . \mathrm{We}$

7 further investigated the two main effects with three post-hoc $t$-tests for each factor. The

8 p-values reported are Bonferroni-adjusted for multiple comparisons.

10 For the main effect of effort, we observed a curved pattern, with completion times

11 lengthening progressively as the effort level increased between $20 \%$ and $60 \%$, before

12 decreasing again slightly for the $80 \%$ effort level (see descriptive statistics in Table 4). The

13 contrasts between adjacent effort levels were all significant (20\% vs $40 \%$ effort:

$14 t(286)=19.7, p<.001, d=1.16 ; 40 \%$ vs $60 \%$ effort: $t(286)=7.07, p<.001, d=0.42$; and

$1560 \%$ vs $80 \%$ effort: $t(286)=8.88, p<.001, d=0.52)$.

17 For the main effect of reward, the descriptive statistics (see Table 4) suggested that completion times decreased slightly with increasing reward level, although the post-hoc comparisons between adjacent reward levels were all non-significant following Bonferroni correction ( 3 vs 6 points: $t(272)=0.08, p=1.0 ; 6$ vs 9 points: $t(272)=1.11, p=.80 ; 9$ vs 12

21 points: $t(272)=2.32, p=.06)$.

23 Finally, in a further, exploratory analysis we also examined whether there was a significant

24 association between participants' maximum allowed time and the six questionnaire measures; 25 however, none of these were significant (all $p$ s $>.05$ ). 
Table 4. Number Switching Task: Descriptive statistics for proportional completion time (across reward and effort levels).

\section{Proportional Completion Time}

\begin{tabular}{|c|c|c|}
\hline Reward (points) & $\mathbf{N}$ & Mean (SD) \\
\hline 3 & 273 & $0.84(0.06)$ \\
\hline 6 & 273 & $0.84(0.05)$ \\
\hline 9 & 273 & $0.84(0.05)$ \\
\hline 12 & 273 & $0.83(0.05)$ \\
\hline \multicolumn{3}{|l|}{ Effort Level } \\
\hline $20 \%$ & 287 & $0.80(0.07)$ \\
\hline $40 \%$ & 287 & $0.85(0.06)$ \\
\hline $60 \%$ & 287 & $0.86(0.05)$ \\
\hline $80 \%$ & 287 & $0.84(0.05)$ \\
\hline
\end{tabular}

1

2 Notes. Times are expressed as a proportion of each participant's maximum allowed completion time.

3 These data only include complete cases, i.e. where participants recorded at least one trial for each level of

4 reward or points.

5

6 


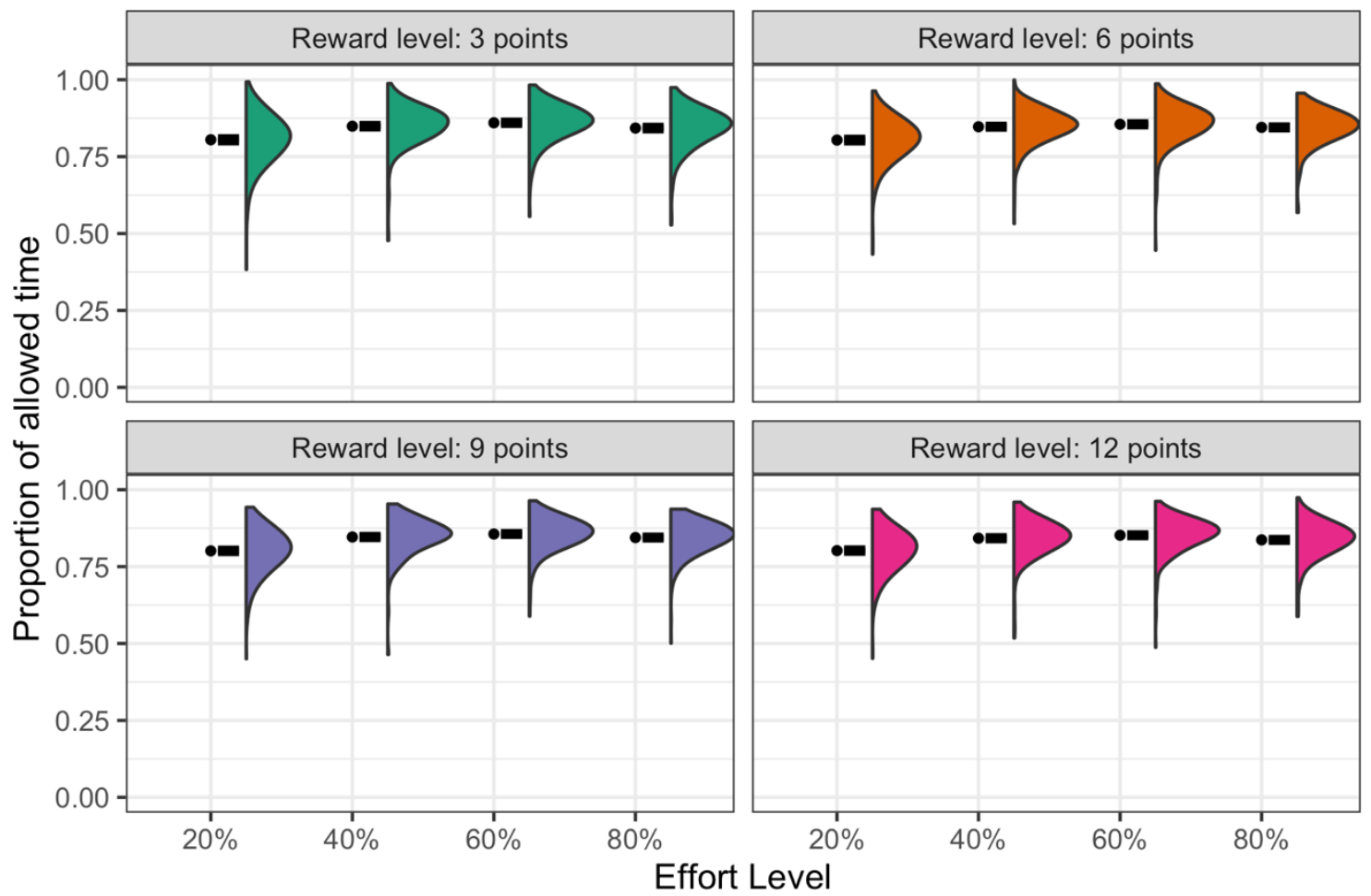

Figure 5. Number Switching Task: completion time. Figure shows the mean, standard error and distribution of the completion times (expressed as a proportion of each participant's allowed time) for each level of reward and effort level. See also Figure S9 for the same plot without faceting.

\section{Subjective Task Load}

5 Participants' ratings of the subjective demand of each effort level are shown in Figure 6, with

6 each scale of the index plotted in a separate panel. Participants reported that they found each

7 effort level successively more demanding, which was confirmed statistically (all ANOVAs

8 indicated a significant effect of effort, $p \mathrm{~s}<.0001)$. Post-hoc $t$-tests of the differences between

9 sequential levels of effort are reported in Table 5. These comparisons were all significant

10 (after Bonferroni correction), except for one: the comparison between ratings of perceived

11 performance on the $60 \%$ and $80 \%$ effort. 
Table 5. Subjective Task Load: post-hoc $t$-tests and standardised effect sizes.

\begin{tabular}{|c|c|c|c|c|c|c|c|c|c|}
\hline & \multicolumn{7}{|c|}{ Effort Level Comparisons } \\
\hline & \multicolumn{2}{|c|}{$20 \%$ vs 40\% } & \multicolumn{2}{c|}{$40 \%$ vs 60\% } & \multicolumn{3}{c|}{$60 \%$ vs 80\% } \\
\hline \multirow{2}{*}{ Mental } & 10.2 & $<.001$ & 0.60 & 10.1 & $<.001$ & 0.59 & 5.65 & $<.001$ & 0.33 \\
Demand & & & & & & & & & \\
\hline Physical & 7.75 & $<.001$ & 0.46 & 5.45 & $<.001$ & 0.32 & 3.46 & .002 & 0.20 \\
Demand & & & & & & & & & \\
\hline Temporal & 4.50 & $<.001$ & 0.26 & 6.26 & $<.001$ & 0.37 & 7.15 & $<.001$ & 0.42 \\
Demand & & & & & & & & & \\
\hline Performance & 2.48 & .04 & 0.15 & 4.02 & $<.001$ & 0.24 & 1.96 & 0.15 & 0.12 \\
\hline Effort & 8.38 & $<.001$ & 0.49 & 5.03 & $<.001$ & 0.30 & 5.52 & $<.001$ & 0.32 \\
\hline Frustration & 6.13 & $<.001$ & 0.36 & 5.05 & $<.001$ & 0.30 & 2.93 & .01 & 0.17 \\
\hline
\end{tabular}

1

2 Note. P-values above are corrected for three multiple comparisons within each scale of the index. Degrees of

3 freedom are 289 throughout. 

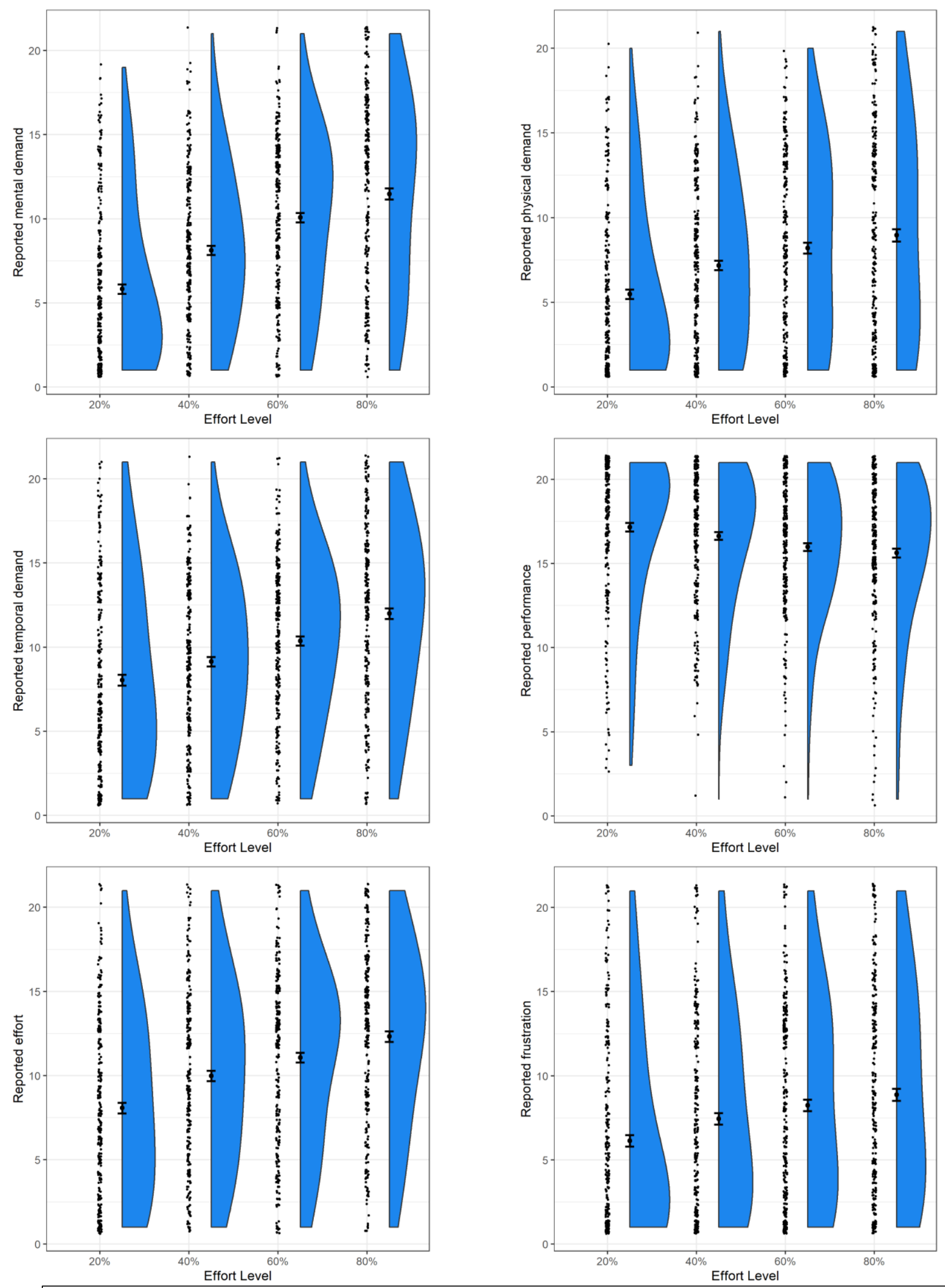

Figure 6. Subjective task load ratings for each effort level. Plots show (from left to right within each plot) the individual data points, the means and standard errors and the distributions of scores for each of the six scales of the index. 
1 Exploratory Analyses

\section{Model Comparison}

3 We started by comparing the twelve models using the Widely-Applicable Information

4 Criterion (WAIC; Watanabe, 2010). WAIC estimates the out-of-sample predictive accuracy

5 of a model, providing both a point estimate and standard error, enabling us to quantify

6 uncertainty.

7

8 The WAIC values for the three best scoring models are plotted in Figure 7. The best model

9 was Model $9\left(\mathrm{vI}+\mathrm{vR}+\mathrm{v} R^{2}+\mathrm{vE}\right)$, so we proceeded with the rest of the modelling analysis

10 using the posterior estimates from this model. The distance to the next two models, Model 4

$11(\mathrm{vI}+\mathrm{vR}+\mathrm{vE})$ and Model $7\left(\mathrm{vI}+\mathrm{vR}+\mathrm{vE}+\mathrm{v} E^{2}\right)$ was fairly large but not definitive (3.2 and

123.3 standard errors respectively), so we also conducted sensitivity analyses using these two

13 models, which are reported in the Supplement. There were no major differences between the

14 inferences drawn from any of these models.

15

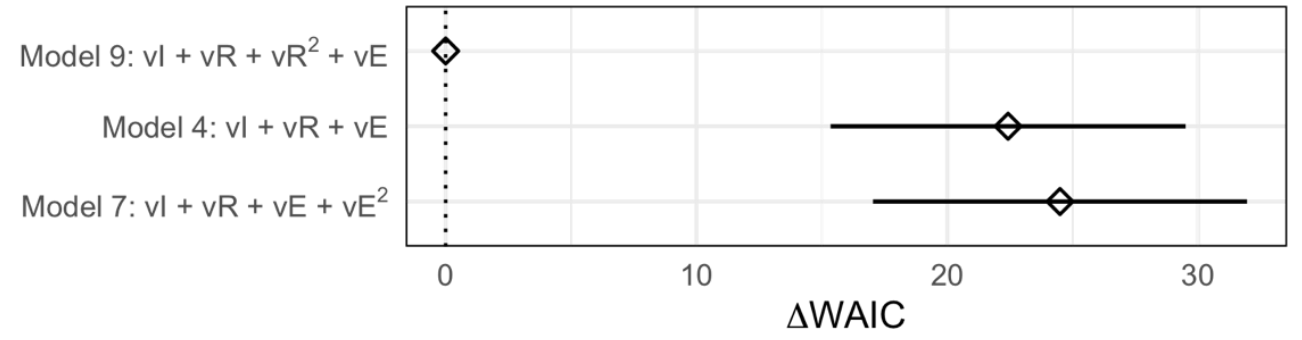

Figure 7. Differences in WAIC relative to the best performing model (Model 9). Also shown is the standard error of this difference (black intervals). For simplicity, only the three best scoring models are shown above; the full plot of all eight models is given in Figure S6. Model 9, the best performing model, contained a varying intercept, varying linear effects of reward and effort and a varying quadratic effect of effort. 
1 Figure 8 shows the posterior estimates of the population-level intercept, reward and effort

2 sensitivity parameters (mean and standard deviation). Note that none of these overlap zero,

3 indicating that, as we would expect, participants are sensitive to both reward and effort (in

4 accordance with the earlier ANOVA results).

5

6

7

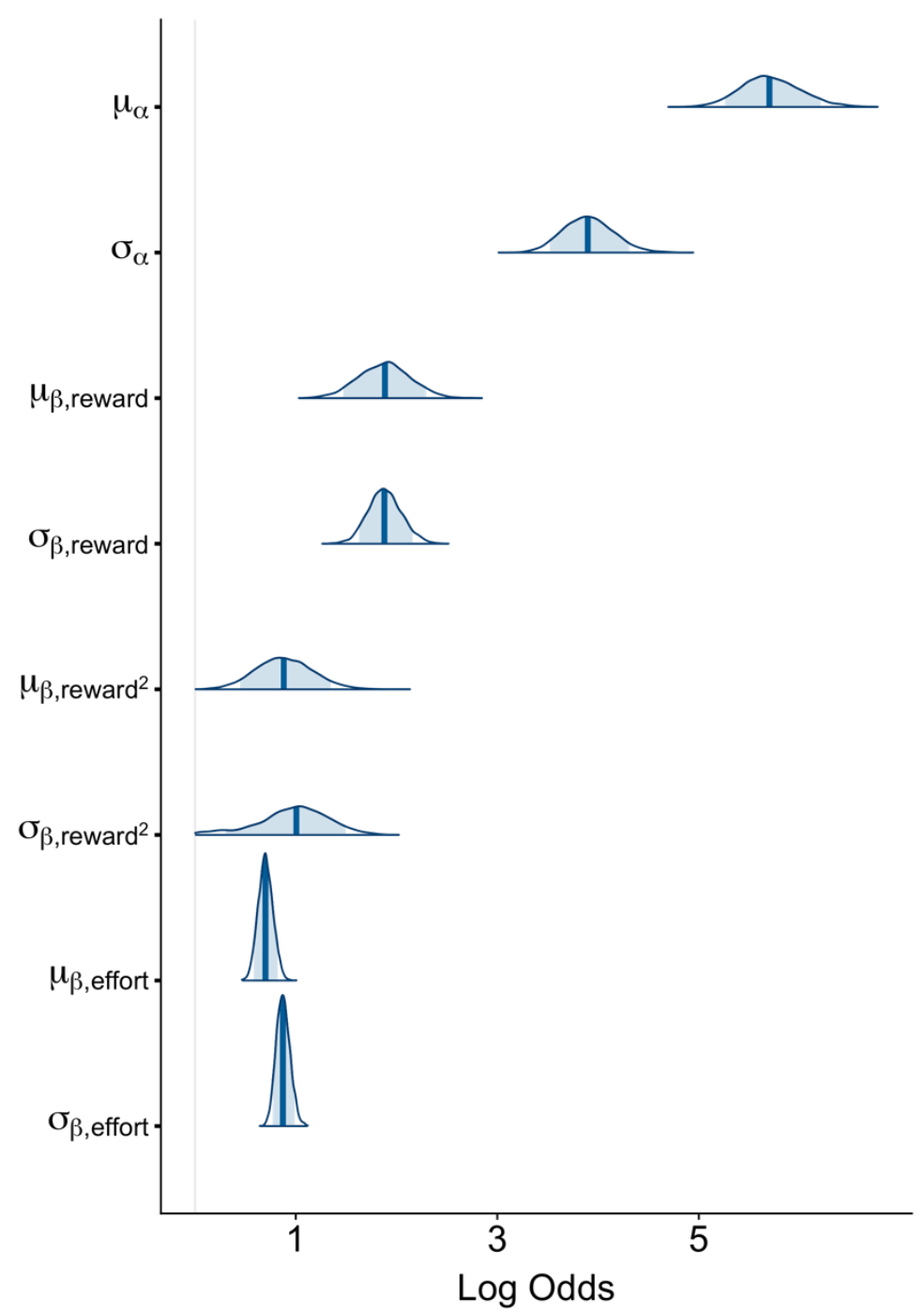

Figure 8. Posterior distributions of the population level parameters from Model $9(v I+v R+$ $v R^{2}+v E$ ). The vertical line indicates the mean of each distribution and the shaded region the $89 \%$ quantile interval. 
1 The posterior predictions of the model are shown in Figure S7. First, we see that the model

2 predicts that the probability of accepting a challenge will decrease as a concave function of

3 effort level and that this decline will be progressively shallower at higher levels of offered

4 reward. This means that this model is able to reproduce not just the basic discounting of

5 reward by effort, but also the specific shape of the discounting curves observed in the data.

6 Secondly, the models also clearly show substantial uncertainty about the exact relationship

7 between the probability of accepting a challenge and effort when it comes to predicting

8 individual participant behaviour. In other words, the average population-level effect is clear

9 but the model implies there is substantial variability between individuals.

10

11 Next, we examined the correlation between the participant-level effort sensitivity parameters

12 and the probability of success on the task (see Figure 9). Importantly, this was not

13 significantly different from zero, $r(288)=0.10, p=.09$, suggesting effort sensitivity was not

14 confounded by probability discounting. 


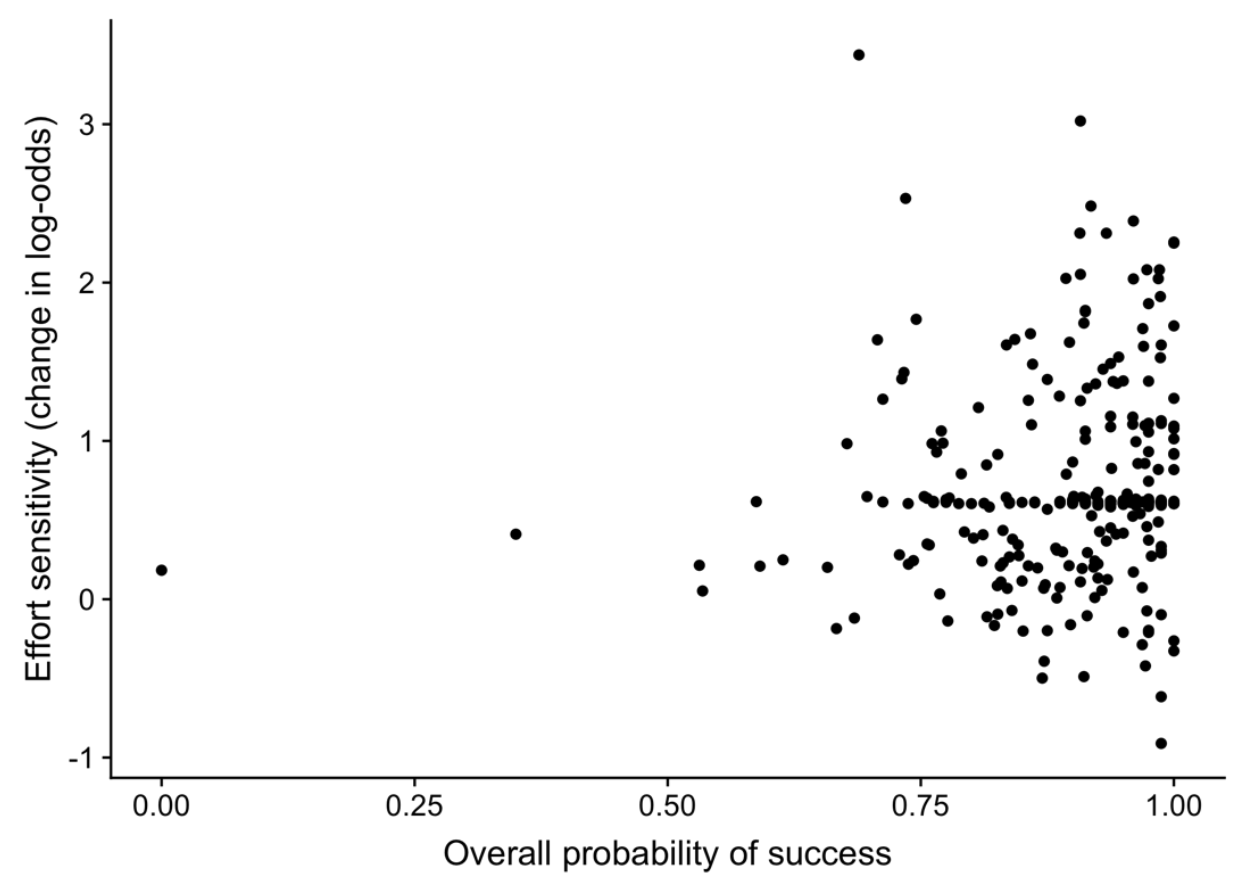

Figure 9. Relationship between the probability of success and effort sensitivity. The correlation was non-significant $(p=.09$ ), implying that effort sensitivity is not confounded by probability discounting in this task. Note the extreme point on the left of the graph corresponds to a participant who accepted (and failed) only one trial overall. In a sensitivity analysis, removing this participant increased the $p$-value of the correlation to $p=.14$.

1

\section{Association of computational parameters with questionnaire measures}

4 The purpose of this final stage of our analysis was to explore any associations between the

5 traits assessed by our questionnaires and the subject-level parameters estimated in Model 9

6 above $\left(\mathrm{vI}+\mathrm{vR}+\mathrm{v} R^{2}+\mathrm{vE}\right)$.

7

8 As an exploratory step we first conducted a series of regressions looking at each

9 questionnaire individually (controlling for age and education each time). We found

10 significant associations between effort sensitivity and Cognitive Complaints $(\beta=0.02$,

$11 p=.04)$, Fatigue Symptoms $(\beta=0.01, p=.04)$, Need for Cognition $(\beta=-0.02, p=.02)$, and

12 Zung Depression Score $(\beta=0.01, p=.04)$. The remaining associations were non-significant: 
1 Experience of Pleasure $(p=.90)$, and Physical Activity $(p=.53)$. With regards to linear

2 reward sensitivity we found an association with Need for Cognition $(\beta=0.04, p=.03)$, as

3 well as with Physical Activity $(\beta=-0.17, p=.03)$, but not the other questionnaire measures.

4 Finally, there were no significant associations with any of the questionnaires for either

5 quadratic reward sensitivity or the intercept parameter. Note we have not corrected for

6 multiple comparisons; we therefore emphasise that the above results should be treated as

7 indicative and exploratory only.

9 To mitigate the multiple comparisons problem and provide a complementary means of 10 assessing these associations, we had also planned a structural equation modelling analysis -

11 this allowed us to test all of the associations at the same time, therefore without requiring

12 correction. First, we used confirmatory factor analysis to compare several possible factor

13 structures which were devised a priori. The CFA is described in detail in the Supplement;

14 briefly, the four structures considered were:

15 - One with a distinct latent factor for each questionnaire

- Another in which the all questions mapped onto a single latent factor, equivalent to a 'P' factor in psychiatry (Caspi et al., 2013)

- A structure in which they were grouped by broad cognitive domain

- Another in which the questionnaires directly relevant to mental health symptoms were grouped together

22 We found the 'full' structure (i.e. with a distinct latent factor for each questionnaire) fitted the 23 data best. 
1 We then conducted an SEM, using this full factor structure as the measurement model, to

2 predict the subject-level intercept, reward and effort sensitivity parameters obtained from

3 Model $9\left(v I+v R+v R^{2}+v E\right)$ of the Number Switching Task. This time we found a

4 significant positive association between Need for Cognition and both the linear and quadratic

5 reward sensitivity parameters (standardised $\beta=0.29, p=.003$, and $\beta=0.06, p=.03$

6 respectively). However, all other associations were non-significant. A possible reason for the

7 discrepancy between this result and those from the simple regressions detailed earlier is that

8 the SEM regression measures the unique variance explained by each latent factor, whereas in

9 fact several of the factors may be making a shared contribution to effort sensitivity. One clue

10 that this is the case is that, in the SEM, the latent factors for cognitive complaints, fatigue,

11 need for cognition and depression symptoms all significantly covaried with one another. Full

12 results from the SEM regression are provided in Tables S5 and S6.

\section{Ruling out temporal discounting effects}

15 One possible issue with the NST is that, because participants were allowed different times in which to complete the sequences, they may experience different rates of reward, in turn introducing temporal discounting effects. To test for this, we examined the association between participants' probability of accepting each level and their average time taken to complete that level - if their choices were affected by temporal discounting we would expect to see a negative correlation, with lower probability of accepting an offer as the duration increases. However, there was no significant association, $r=0.002, t(4304)=0.13, p=.89$, suggesting temporal discounting did not affect participants' choices. In a complementary analysis we also tested the correlation between participants' overall tendency to accept (the

24 intercept parameter estimated by Model 9) and their allowed time; again this was non- 
1 significant, $r=-0.03, t(0.53), p=.59$. Further tests of the correlations between allowed time

2 and other variables are included in the Supplement, Table S7. 


\section{Discussion}

2 We have presented a new task, the NST, for measuring cognitive effort and demonstrated that

3 it resolves one of the major shortcomings of existing measures, namely the confounding of

4 effort by task difficulty. In our results, obtained from a large online sample, participants

5 treated higher effort levels as more costly, despite being just as likely to win the offered

6 reward. The idea that cognitive effort is costly is one of the foundational assumptions of

7 cognitive effort research, and is supported by earlier studies showing effort discounting in

8 several contexts (Apps et al., 2015; McLaughlin et al., 2021; Crawford, Eisenstein et al.,

9 2022; Crawford, English et al., 2022; Kool et al., 2010; Kool \& Botvinick, 2014; Westbrook

10 et al., 2013); importantly, however, this is the first time that effort discounting has been

11 studied in a task where effort and difficulty are not confounded. In other words, we were able

12 to manipulate and measure cognitive effort without the problem of probability discounting.

14 A related concern was that we need to be able to standardise the difficulty of the task,

15 otherwise comparisons across participants (for example between patient groups and healthy

16 controls), may not be valid. In the NST this is achieved by tailoring the time allowed for

17 completion of each sequence to participants individually. Encouragingly, the success rates

18 were very consistent across participants, suggesting the standardisation procedure was

19 successful.

21 The finding that completion times generally became longer as the effort level increased is

22 consistent with these levels requiring more cognitive control (and therefore effort; Shenhav et

23 al., 2017), but the small reduction in completion time at the highest level was unexpected.

24 This is mirrored by a similar, though non-significant, increase in accuracy at the highest

25 effort levels. The most likely explanation is that half of the sequences at this level involved 
1 alternating on every digit (i.e. eight switches), which, even though participants could not be

2 sure exactly how many switches they would be shown, may have permitted them to respond

3 slightly faster and/or more accurately. If so, this should be straightforward to address - future

4 iterations of this task could use a ten- rather than nine-digit sequence, so that sequences

5 which alternate on every digit are no longer possible. This would have the further advantage

6 of requiring one digit to be shown twice, making it impossible for participants to work out

7 which digits remain to be shown.

9 A key component of the NST's design is that the time allowed to complete the nine-digit

10 sequences is personalised to each participant. This allows us to normalise the difficulty of the

11 task and adjust for differences between participants in cognitive capacity; however, it also

12 introduces the potential for temporal discounting, since participants may experience different

13 rates of reward. Fortunately we were able to rule out effects of temporal discounting in our

14 results; we found that there was no association between participants' effort sensitivity and the

15 time they were had to complete the sequences. Thus we can be relatively confident that the

16 NST is measuring effort discounting rather than temporal discounting.

18 One last feature of the NST results that deserves comment is the observation that the

19 acceptance rates are relatively high (averaging over 50\%) across all of the conditions. This is

20 by design - the task needed to be suitable for use with patients, who are likely to have much

21 lower acceptance rates than the healthy participants we have initially tested here.

22 Nevertheless, if in future studies the acceptance rates are seen to be too high then a possible

23 change might be, for example, to offer a low reward/low effort alternative during the choice

24 phase, rather than the current accept/reject choice. This could also permit calculation of

25 subjective indifference points if desired. 
1 The Subjective Task Load results show clearly that participants reported finding each level of

2 effort progressively more demanding. The increase in physical demand at higher effort levels

3 is a potential issue, as participants should in theory be making the same number of key

4 presses at approximately the same rate across all levels. This result may be genuine- - for

5 example, the time pressure on the task could be felt as a kind of physical demand, for

6 example causing participants to tense up — but it could also suggest the presence of

7 experimenter demand effects. One way to address this in future would be to modify the

8 language used in the offers - rather than referring to difficulty levels, it may be preferable for

9 the offers to refer to the switching rate explicitly instead (alternatively, abstract shapes or

10 colours could be used to label each effort level, avoiding words entirely). That said, we also

11 found that in post-hoc tests the only nonsignificant difference was on the performance

12 subscale, between the two highest effort levels, which directly matches the behavioural result

13 discussed above, that participants performed slightly faster at $80 \%$ than $60 \%$ effort. That

14 participants were sensitive to this detail gives some reassurance that the Subjective Task

15 Load results were accurate appraisals after all.

17 The remainder of our analyses were exploratory in nature and aimed principally at demonstrating how this task can be used for individual differences research. The most parsimonious model according to WAIC included linear and quadratic effects of reward and a

20 linear effect of effort, all of which varied across participants. However, we should be clear

21 that this model is linear on a log-odds scale only, implying non-linear (specifically convex) effort costs on the outcome scale, consistent with other work (see Ritz et al., 2021). The model also indicated there was substantial variability in effort sensitivity across participants,

24 which will be beneficial for individual differences research. 
1 In a planned analysis, we used SEM to measure the association between several trait

2 measures and the participant-level parameters from the behavioural model. The only

3 significant association was between Need for Cognition, a construct representing

4 participants' enjoyment of cognitively demanding activity, and reward sensitivity, the extent

5 to which participants' choices changed in line with the offered reward. Possibly this is

6 because participants who score higher on Need for Cognition pay more attention to the

7 parameters of the task. While the lack of other associations, particularly with effort

8 sensitivity, was somewhat surprising, one issue may be overlap between the multiple

9 questionnaire measures we included. Ideally this would have been fully accounted for by the

10 factor analysis, but nevertheless we still saw remaining covariance between the latent factors

11 in the SEM. As we mention in the results section above, this covariance may mean that the

12 factors are making a shared contribution to effort sensitivity which is not detected by the

13 SEM (since the latter only measures the unique contribution of each factor).

15 In line with this explanation, when we examined the relationship between each questionnaire

16 and the outcome variables in separate regressions, we found that there were indeed

17 significant associations between effort sensitivity and a number of the mental health related

18 questionnaires (Cognitive Complaints Inventory, Fatigue Severity Scale and Zung

19 Depression Scale). In addition the negative association with Need for Cognition, meaning

20 that participants who enjoy cognitive demanding activity were less sensitive to effort,

21 provides particular reassurance about the external validity of the model. We nevertheless urge caution about not overinterpreting the results of the simple regressions alone; with many tests carried out we unavoidably run into the problem of multiple comparisons, so these results

24 should only be regarded as exploratory for now. 
1 Finally, we are optimistic about the use of the NST in clinical populations, but again more

2 validation will clearly be needed to support this. In addition, further work could be conducted

3 to design more sophisticated models; those presented in this paper represent a starting point,

4 and natural extensions are to explicitly model the corelations between the sensitivity

5 parameters, or add a lapse component that acknowledges that on some trials participants may

6 simply decide at random. Numerous other model variations can be devised and built, and

$7 \quad$ would be interesting topics of study.

8

9 In summary, we have presented a new task measuring cognitive effort, which resolves a

10 longstanding problem of conflating the effort demanded by a task with its difficulty. Not only

11 have we been able to manipulate effort without changing the difficulty of the task, but we can

12 additionally standardise the difficulty across participants by tailoring the time allowed

13 according to performance at an individual level. This is the first cognitive effort task in which

14 such standardisation can be achieved and means individual differences research can be

15 carried out without concerns around confounding from difficulty or ability.

17 Acknowledgements

18 This work was funded by a Wellcome Trust 4-year PhD studentship awarded to HF. OJR was

19 supported by a Medical Research Council senior non-clinical fellowship [MR/R020817/1].

20 Open Practices

21 This study was preregistered on the Open Science Framework

22 (doi:10.17605/OSF.IO/8Y7P9). There were no deviations from this plan. All data and

23 analysis scripts are provided at the Open Science Foundation repository 
1 (doi:10.17605/OSF.IO/X34KN) and code to run the Number Switching Task is deposited in

2 the Gorilla Open Materials Repository (https://app.gorilla.sc/openmaterials/328049).

3

4 Declarations

\section{$5 \quad$ Funding}

6 This work was funded by a Wellcome Trust 4-year PhD studentship awarded to HF. OJR was

7 supported by a Medical Research Council senior non-clinical fellowship [MR/R020817/1].

$8 \quad$ Competing Interests

9 HF has no financial or non-financial interests to declare. OJR's MRC senior fellowship is

10 partially in collaboration with Cambridge Cognition Ltd (who plan to provide in-kind

11 contribution) and he is running an investigator-initiated trial with medication donated by

12 Lundbeck (escitalopram and placebo, no financial contribution). He also holds an MRC-

13 Proximity to discovery award with Roche (who provide in-kind contributions and have

14 sponsored travel for ACP) regarding work on heart-rate variability and anxiety. He has also

15 completed consultancy work on affective bias modification for Peak and online CBT for

16 IESO digital health. OJR sits on the committee of the British Association of

17 Psychopharmacology. In the past 3 years JPR has held a PhD studentship co-funded by

18 Cambridge Cognition and performed consultancy work for GE Ltd. These disclosures are

19 made in the interest of full transparency and do not constitute a conflict of interest with the

20 current work.

21 Ethics Approval

22 This study was approved by the UCL Research Ethics Committee, reference 6198/001, and

23 the study was performed in accordance with the Declaration of Helsinki. 
1 Consent to Participate

2 All participants gave informed consent prior to taking part.

3

4 Consent to Publication

$5 \quad$ All participants consented to publication of their anonymised data prior to taking part.

6

7 Availability of Data, Materials and Code

8 This study was preregistered on the Open Science Framework, doi:10.17605/OSF.IO/8Y7P9.

9 All data and analysis scripts are provided at the Open Science Foundation repository,

10 doi:10.17605/OSF.IO/X34KN, and code to run the Number Switching Task is deposited in

11 the Gorilla Open Materials Repository, https://app.gorilla.sc/openmaterials/328049.

12

13 Authors Contributions

14 HF, OJR and JR contributed to the design of the study. HF collected the data. HF, OJR and

15 JR contributed to the writing of this report.

16

17

18 


\section{References}

2 Allen, M., Poggiali, D., Whitaker, K., Rhys Marshall, T., Kievit, R. (2019). Raincloud plots:

3 A multi-platform tool for robust data visualization. Wellcome Open Research, 4(63), 1-41.

4 doi:10.12688/wellcomeopenres.15191.1.

5

6 Apps, M., Grima, L., Manohar, S., \& Husain, M. (2015). The role of cognitive effort in

7 subjective reward devaluation and risky decision-making. Sci Rep, 5, 16880.

8 doi:10.1038/srep16880.

9

10 Bates D., Mächler M., Bolker B., Walker S. (2015). Fitting Linear Mixed-Effects Models

11 Using lme4. Journal of Statistical Software, 67(1), 1-48. doi:10.18637/jss.v067.i01.

13 Bays, P., Catalao, R., \& Husain, M. (2009). The precision of working memory is set by

14 allocation of a shared resource. $J o V, 9(10), 1-11$. doi:10.1167/9.10.7.

16 Betancourt, M. (2018). A Conceptual Introduction to Hamiltonian Monte Carlo.

17 ArXiv:1701.02434. https://arxiv.org/abs/1701.02434.

18

19 Bonnelle, V., Manohar, S., Behrens, T., \& Husain, M. (2016). Individual differences in

20 premotor brain systems underlie behavioural apathy. Cerebral Cortex, 26, 807-819.

22 Braver, T. (2012). The variable nature of cognitive control: A dual mechanisms framework.

23 Trends in Cognitive Science, 16, 106-113. doi:10.1016/j.tics.2011.12.010. 
1 Caspi, A., Houts, R., Belsky, D., Goldman-Mellor, S., Harrington, H., Israel, S., Meier, M.,

2 Ramrakha, S., Shalev, I., Poulton, R., \& Moffitt, T. (2013). The p Factor: One General

3 Psychopathology Factor in the Structure of Psychiatric Disorders? Clin Psych Sci, 2(2).

4 doi:10.1177/2167702613497473.

5

6 Chong, T., Bonnelle, J., \& Husain, M. (2016). Quantifying motivation with effort-based

7 decision-making paradigms in health and disease. Progress in Brain Research, 229, 71-100.

8 doi:10.1016/bs/pbr.2016.05.002.

9

10 Coelho, G., Hanel, P., Wolf, L. (2018). The very efficient assessment of Need for Cognition:

11 Developing a six-item version. Assessment, 27(8), 1870-1885. doi:10.1177/1073191118793208.

14 Crawford, J., Eisenstein, S., Peelle, J., \& Braver, T. (2022). Domain general cognitive 15 motivation: Evidence from economic decision-making - Final Registered Report. Cognitive 16 Research: Principles and Implications, 7, 23. doi:10.1186/s41235-022-00363-z.

Crawford, J., English, T., \& Braver, T. (2022). Cognitive effort-based decision-making 19 across experimental and daily life indices in younger and older adults. The Journals of 20 Gerontology, Series B, 27(8), 1870-1885. doi:10.1093/geronb/gbac167.

22 Gabry, J. \& Češnovar, R. (2020). cmdstanr: R Interface to 'CmdStan'. https://mcstan.org/cmdstanr, https://discourse.mc-stan.org 
1 Gard, D., Gard, M., Kring, A., John, O. (2006). Anticipatory and consummatory components

2 of the experience of pleasure: A scale development study. Journal of Research in

3 Personality, 40, 1086-1102. doi:10.1016/j.jrp/2005.11.001.

4

5 Gold, J., Waltz, J., \& Frank, M. (2015). Effort cost computation in schizophrenia: A

6 commentary on the recent literature. Biological Psychiatry, 78(11).

7 doi:10.1016/j.biopsych.2015.05.005.

8

9 Hart, S., \& Staveland, L. (1988). Development of NASA-TLX (Task Load Index): Results of 10 Empirical and Theoretical Research. Advances in Psychology, 52, 139-183.

11 doi:10.1016/S0166-4115(08)62386-9.

12

13 Husain, M. \& Roiser, J. (2018). Neuroscience of apathy and anhedonia: A transdiagnostic 14 approach. Nat Rev Neurosci 19, 470-484. doi:10.1038/s41583-018-0029-9.

16 Iverson, G., \& Lam, R. (2013). Rapid screening for perceived cognitive impairment in major 17 depressive disorder. Ann Clin Psychiatry, 25(2), 135-140. PMID: 23638444.

19 Kassambara, A. (2020). rstatix: Pipe-Friendly Framework for Basic Statistical Tests. R

20 package version 0.6.0. https://CRAN.R-project.org/package=rstatix

21

22 Kahneman, D. (1973). Attention and Effort. Prentice Hall, NJ, USA. Accessed at: http://citeseerx.ist.psu.edu/viewdoc/download?doi=10.1.1.398.5285\&rep=rep1\&type=pdf 
1 Krupp, L., LaRocca, N., Muir-Nash, J., \& Steinberg, A. (1989). The Fatigue Severity Scale:

2 Application to Patients with Multiple Sclerosis and Systemic Lupus Erythematosus. Arch 3 Neurol, 46(10), 1121-1123.

4

5 Lee, P., Macfarlane, D., Lam, T., \& Stewart, S. (2011). Validity of the International Physical

6 Activity Questionnaire Short Form (IPAQ-SF): a systematic review. Int. J. Behav. Nutr.

$7 \quad$ Phys. Act., 8(115), 1-11. doi:10.1001/archneur.1989.00520460115022.

8

9 McElreath, R. (2020) Statistical Rethinking. Chapman and Hall/CRC.

10

11 McGuire, J., Botvinick, M. (2010). Prefrontal cortex, cognitive control, and the registration

12 of decision costs. Proc. Natl. Acad. Sci. U.S.A. 107, 7922-7926.

13 doi:10.1073/pnas.0910662107.

14

15 McLaughlin, D., Braver, T., \& Peelle, J. (2021). Measuring the subjective cost of listening 16 effort using a discounting task. Journal of Speech, Language and Hearing Research. 64(2), 17 337-347. doi:10.1044/2020_JSLHR-20-00086.

19 Mesholam-Gately, R., Giuliano, A., Goff, K., Faraone, S., \& Seidman, L. (2009).

20 Neurocognition in first-episode schizophrenia: A meta-analytic review. Neuropsychology 21 23(3), 315-336. doi:10.1037/a0014708.

23 Ortega, P., Braun, D., Dyer, J., Kim, K., Tishby, N. (2015). Information-Theoretic Bounded 24 Rationality. ArXiv:1512.06789. https://arxiv.org/abs/1512.06789 
1 Ritz, H., Leng, X., \& Shenhav, A. (2021). Cognitive control as a multivariate optimisation

2 problem. ArXiv:2110.00668. https://arxiv.org/abs/2110.00668

3

4 Rock, P., Roiser, J., Riedel, W., \& Blackwell, A. (2014). Cognitive impairment in depression:

5 A systematic review and meta-analysis. Psychol Med, 44(10), 2029-2040.

6 doi:10.1017/s0033291713002535.

7

8 Rosseel, Y. (2012). lavaan: An R Package for Structural Equation Modelling. Journal of

$9 \quad$ Statistical Software, 48(2), 1-36. doi:10.18637/jss.v048.i02.

10

11 R Core Team (2019). R: A language and environment for statistical computing. R Foundation for Statistical Computing, Vienna, Austria. https://www.R-project.org/

14 Schmidt, L., Lebreton, M., Clery-Melin, M.-L., Daunizeau, J., Pessiglione, M. (2012). Neural 15 mechanisms underlying motivation of mental versus physical effort. PLoS Biol., 10(2).

16 doi:10.1371/journal.pbio.1001266.

17

Shenhav, A., Botvinick, M., \& Cohen, J. (2013). The Expected Value of Control: An

Integrative Theory of Anterior Cingulate Cortex Function. Neuron, 79(2), 217 - 240. doi:10.1016/j.neuron.2013.07.007

21

22 Shenhav, A., Musslick, S., Lieder, F., Kool, W., Griffiths, T., Cohen, J., \& Botvinick, M.

23 (2017). Toward a Rational and Mechanistic Account of Mental Effort. Annu. Rev. Neurosci.

24 40, 99-124. doi:10.1146/annurev-neuro-072116-031526. 
1 Stan Development Team. (2021). Stan Modelling Language Users Guide and Reference

2 Manual. https://mc-stan.org

3

4 Toplak, M., West, R., \& Stanovich, K. (2011). The Cognitive Reflection Test as a predictor 5 of performance on heuristics-and-biases tasks. Memory and Cognition, 39, 1275 - 1289.

6 doi:10.3758/s13421-011-0104-1.

7

8 Watanabe, S. (2010). Asymptotic equivalence of Bayes cross validation and widely

9 application information criterion in singular learning theory. Journal of Machine Learning

10 Research, 11, 3571-3594. doi:10.5555/1756006.1953045.

11

12 Westbrook, A., \& Braver, T. (2015). Cognitive effort: A neuroeconomic approach. Cogn.

13 Affect. Behav. Neurosci., 15, 395-415. doi:10.3758/s13415-015-0334-y.

14

15 Westbrook, A., Kester, D., \& Braver, T. (2013). What is the subjective cost of cognitive

16 effort? Load, trait and ageing effects revealed by economic preference. PLoS One, 8(7), 1-8.

17 doi:10.1371/journal.pone.0068210.

18

19 Zung, W. (1965). A Self-Rating Depression Scale. Arch Gen Psychiatry, 12, 63-70.

20 doi:10.1001/archpsyc.1965.01720310065008.

21

22 


\section{Supplementary Materials}

2

3

4

\section{Method}

\section{Questionnaires}

NASA Task Load Index. The NASA Task Load Index (Hart \& Staveland, 1988), referred to in the main text as the 'Subjective Task Load', assesses subjective workload on six subscales: mental demand, physical demand, temporal demand, performance, effort and frustration. For each subscale and, in our case, for each level of effort, participants were asked to rate their experience of the task on a 21-point scale from 'very low' to 'very high'. The six subscales were presented in the same order as above, within which the questions about the different effort levels were randomised. We report participants' scores on each subscale for each effort level separately.

Cognitive Complaints Inventory. The Cognitive Complaints Inventory (Iverson \& Lam, 2013) is a six-item questionnaire in which participants rate their problems with concentration, memory and thinking skills, on a four-point scale from 0 (not at all) to 3 (very much). We report total scores (where higher scores indicate more cognitive complaints).

We appended a catch question (“Select 'very much' for this question") to this questionnaire to identify participants who were not paying attention. We placed this at the end to avoid interfering with the psychometric properties of the questionnaire itself.

Fatigue Severity Scale. The Fatigue Severity Scale (Krupp, LaRocca, Muir-Nash \& Steinberg, 1989) is a nine-item questionnaire in which participants rate their experience of 
1 fatigue and the impact fatigue has on their daily activities, on a scale from 1 to 7 . We report

2 total scores (where higher scores indicate more fatigue).

$4 \quad$ International Physical Activity Questionnaire Short Form. The International Physical

5 Activity Questionnaire Short Form (IPAQ-SF; Lee, Macfarlane, Lam \& Stewart, 2011) is a

6 seven-item scale that measures self-assessed physical activity over the previous seven days.

7 Participants are asked on how many days and on average for how long each day they spent

8 engaged in vigorous activity, moderate activity, walking and sitting. These estimates are

9 weighted by their estimated metabolic requirements and summed to generate an overall score

10 (termed 'MET-minutes', a way of expressing activity relative to a resting metabolic rate), as

11 follows:

- If necessary, bouts of activity are truncated at a maximum of three hours.

- Walking MET-minutes per week $=3.3 *$ walking minutes $*$ walking days

- $\quad$ Moderate MET-minutes per week $=4 *$ walking minutes $*$ walking days

- Vigorous MET-minutes per week $=8 *$ walking minutes * walking days

- $\quad$ Total MET-minutes per week = Walking Met-minutes + Moderate Met-minutes + Vigorous Met-minutes

Need for Cognition Scale (6-item version). The six-item Need for Cognition Scale (Coelho, Hanel \& Wolf, 2018) measures the extent to which participants enjoy engaging in difficult cognitive activity. Participants rate each of six statements from 1 (not characteristic of themselves) to 5 (characteristic). We report participants' total scores (where higher scores indicate greater enjoyment of cognitively demanding activity).

We added another catch question to the end of this questionnaire. 
1 Temporal Experience of Pleasure Scale. The Temporal Experience of Pleasure Scale (TEPS;

2 Gard, Gard, Kling \& John, 2006) is an 18-item scale with two subscales: a 10-item

3 anticipatory pleasure scale and an 8-item consummatory scale. Each item consists of a

4 statement (e.g. "The smell of freshly cut grass is enjoyable to me") which participants rate on

5 a 6-point scale from 'very false for me' to 'very true for me'. We report total scores (where

6 higher scores indicate greater disposition to experience of pleasure or, equivalently, lower

7 anhedonia).

8

9 Zung Depression Scale. The Zung Depression Scale (Zung, 1965) is a 20-item questionnaire

10 in which participants respond to a series of statements about how they might feel on a 4-point

11 scale from 'a little of the time' to 'most of the time'. We report total scores (where higher

12 scores indicate more depressive symptoms).

14 Computational Models

15 Full specification of models

16 Model 1. Varying Intercept

17 Equation $\mathrm{S1}$

$Y_{\text {subject,trial }} \sim \operatorname{Bernoulli}\left(p_{\text {subject }}\right)$

$$
p_{\text {subject }}=\operatorname{logistic}\left(\alpha_{\text {subject }}\right)
$$

$\alpha_{\text {subject }} \sim \operatorname{Normal}\left(\mu_{\alpha}, \sigma_{\alpha}\right)$

$\mu_{\alpha} \sim \operatorname{Normal}(0,1.5)$

$\sigma_{\alpha} \sim$ Exponential(2) 
$1 \quad$ Model 2. Fixed Intercept and Fixed Linear Effects of Reward and Effort

2 Equation $S 2$

9 Model 3. Varying Intercept and Fixed Linear Effects of Reward and Effort

\section{Equation $S 3$}

$$
Y_{\text {subject,trial }} \sim \operatorname{Bernoulli}\left(p_{\text {trial }}\right)
$$

$$
p_{\text {trial }}=\operatorname{logistic}\left(\alpha+\beta_{\text {reward }} \text { reward }_{\text {trial }}+\beta_{\text {effort }} \text { effort } \text { trial }\right)
$$

$$
\alpha \sim \operatorname{Normal}(0,1.5)
$$

$$
\begin{aligned}
& \beta_{\text {reward }} \sim \operatorname{Normal}(0,1) \\
& \beta_{\text {effort }} \sim \operatorname{Normal}(0,1)
\end{aligned}
$$

Model 4. Varying Intercept and Varying Linear Effects of Reward and Effort

Equation S4

$$
\begin{gathered}
Y_{\text {subject,trial }} \sim \operatorname{Bernoulli}\left(p_{\text {subject,trial }}\right) \\
p_{\text {subject,trial }}=\operatorname{logistic}\left(\alpha_{\text {subject }}+\beta_{\text {reward,subject }} \operatorname{reward}_{\text {trial }}+\beta_{\text {effort,subject }} \text { effort }_{\text {trial }}\right) \\
\alpha_{\text {subject }} \sim \operatorname{Normal}\left(\mu_{\alpha}, \sigma_{\alpha}\right) \\
\mu_{\alpha} \sim \operatorname{Normal}(0,1.5)
\end{gathered}
$$


$9 \quad$ Model 5. Fixed Intercept, Fixed Linear Effect of Reward and Fixed Linear and Quadratic

10 Effects of Effort

11 Equation S5

12

13

14

15

16

17

18

19

20

21

22

23

$$
\sigma_{\alpha} \sim \text { Exponential(2) }
$$

$$
\beta_{\text {reward,subject }} \sim \operatorname{Normal}\left(\mu_{\text {reward }}, \sigma_{\text {reward }}\right)
$$$$
\mu_{\text {reward }} \sim \operatorname{Normal}(0,1)
$$$$
\sigma_{\text {reward }} \sim \text { Exponential(2) }
$$

$$
\beta_{\text {effort }, \text { subject }} \sim \operatorname{Normal}\left(\mu_{\text {effort }}, \sigma_{\text {effort }}\right)
$$

$$
\mu_{\text {effort }} \sim \operatorname{Normal}(0,1)
$$

$$
\sigma_{\text {effort }} \sim \text { Exponential(2) }
$$

$$
Y_{\text {subject,trial }} \sim \operatorname{Bernoulli}\left(p_{\text {trial }}\right)
$$

$$
p_{\text {trial }}=\operatorname{logistic}\left(\alpha+\beta_{\text {reward }} \text { reward }_{\text {trial }}+\beta_{\text {effort }} \text { effort }\right. \text { trial }
$$

$$
+\beta_{\text {quad_effort }} \text { effort } \text { trial }^{2} \text { ) }
$$

$$
\alpha \sim \operatorname{Normal}(0,1.5)
$$

$$
\beta_{\text {reward }} \sim \operatorname{Normal}(0,1)
$$$$
\beta_{\text {effort }} \sim \operatorname{Normal}(0,1)
$$

$$
\beta_{\text {quad_effort }} \sim \operatorname{Normal}(0,1)
$$


1 Model 6. Varying Intercept, Fixed Linear Effect of Reward and Fixed Linear and Quadratic

2 Effects of Effort

3 Equation S6

4

5

6

7

8

10

11

12

13

14 Model 7. Varying Intercept, Varying Linear Effect of Reward and Varying Linear and

15

16

17

18

19

20

21

22

23

$$
Y_{\text {subject,trial }} \sim \operatorname{Bernoulli}\left(p_{\text {subject,trial }}\right)
$$

$$
p_{\text {subject,trial }}=\operatorname{logistic}\left(\alpha_{\text {subject }}+\beta_{\text {reward }} \text { reward }_{\text {trial }}+\beta_{\text {effort }} \text { effort } \text { trial }_{\text {f }}\right.
$$$$
+\beta_{\text {quad_effort }} \text { effort } \text { trial }^{2} \text { ) }
$$

$$
\alpha_{\text {subject }} \sim \operatorname{Normal}\left(\mu_{\alpha}, \sigma_{\alpha}\right)
$$$$
\mu_{\alpha} \sim \operatorname{Normal}(0,1.5)
$$$$
\sigma_{\alpha} \sim \text { Exponential(2) }
$$

$$
\beta_{\text {reward }} \sim \operatorname{Normal}(0,1)
$$$$
\beta_{\text {effort }} \sim \operatorname{Normal}(0,1)
$$

$$
\beta_{\text {quad_effort }} \sim \operatorname{Normal}(0,1)
$$

\section{Quadratic Effects of Effort}

\section{Equation S7}

$$
Y_{\text {subject,trial }} \sim \operatorname{Bernoulli}\left(p_{\text {subject,trial }}\right)
$$

$p_{\text {subject,trial }}=\operatorname{logistic}\left(\alpha_{\text {subject }}+\beta_{\text {reward,subject }}\right.$ reward $_{\text {trial }}+\beta_{\text {effort,subject }}$ effort trial

$$
+\beta_{\text {quad_effort,subject }} \text { effort } \text { trial }^{2} \text { ) }
$$

$$
\alpha_{\text {subject }} \sim \operatorname{Normal}\left(\mu_{\alpha}, \sigma_{\alpha}\right)
$$

$$
\mu_{\alpha} \sim \operatorname{Normal}(0,1.5)
$$

$$
\sigma_{\alpha} \sim \text { Exponential(2) }
$$

$$
\beta_{\text {reward,subject }} \sim \operatorname{Normal}\left(\mu_{\text {reward }}, \sigma_{\text {reward }}\right)
$$

$$
\mu_{\text {reward }} \sim \operatorname{Normal}(0,1)
$$




$$
\sigma_{\text {reward }} \sim \text { Exponential }(2)
$$

$$
\beta_{\text {effort,subject }} \sim \operatorname{Normal}\left(\mu_{\text {effort }}, \sigma_{\text {effort }}\right)
$$

$$
\mu_{\text {effort }} \sim \operatorname{Normal}(0,1)
$$

$$
\sigma_{\text {effort }} \sim \text { Exponential(2) }
$$

$$
\beta_{\text {quad_effort,subject }} \sim \operatorname{Normal}\left(\mu_{\text {quad_effort }}, \sigma_{\text {quad_effort }}\right)
$$

$$
\mu_{\text {quad_effort }} \sim \operatorname{Normal}(0,1)
$$

$\sigma_{\text {quad_effort }} \sim$ Exponential(2)

8

Model 8. Varying Intercept, Varying Linear Effect of Reward and Varying Quadratic Effect of Effort

Equation 58

$$
\begin{gathered}
Y_{\text {subject,trial }} \sim \operatorname{Bernoulli}\left(p_{\text {subject,trial }}\right) \\
p_{\text {subject,trial }}=\operatorname{logistic}\left(\alpha_{\text {subject }}+\beta_{\text {reward,subject }} \text { reward }_{\text {trial }}\right. \\
\left.+\beta_{\text {quad_effort,subject }} \text { effort }_{\text {trial }}{ }^{2}\right) \\
\alpha_{\text {subject }} \sim \operatorname{Normal}\left(\mu_{\alpha}, \sigma_{\alpha}\right) \\
\mu_{\alpha} \sim \operatorname{Normal}(0,1.5) \\
\sigma_{\alpha} \sim \text { Exponential }(2)
\end{gathered}
$$$$
\beta_{\text {reward,subject }} \sim \operatorname{Normal}\left(\mu_{\text {reward }}, \sigma_{\text {reward }}\right)
$$$$
\mu_{\text {reward }} \sim \operatorname{Normal}(0,1)
$$$$
\sigma_{\text {reward }} \sim \text { Exponential(2) }
$$

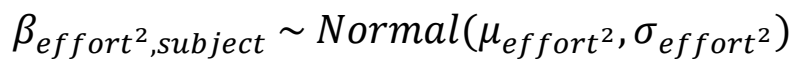$$
\mu_{\text {effort }}^{2} \sim \operatorname{Normal}(0,1)
$$

$$
\sigma_{\text {effort }}^{2} \sim \text { Exponential(2) }
$$


1 Model 9. Varying Intercept, Varying Linear and Quadratic Effects of Reward and Varying

2 Linear Effect of Effort

3 Equation $S 9$

4

$$
Y_{\text {subject,trial }} \sim \operatorname{Bernoulli}\left(p_{\text {subject,trial }}\right)
$$

$$
p_{\text {subject,trial }}=\operatorname{logistic}\left(\alpha_{\text {subject }}+\beta_{\text {reward,subject }} \text { reward }_{\text {trial }}\right.
$$$$
\left.+\beta_{\text {quad_reward,subject }} \text { reward } \text { trial }^{2}+\beta_{\text {effort,subject }} \text { effort } \text { trial }_{\text {frial }}\right)
$$

$$
\alpha_{\text {subject }} \sim \operatorname{Normal}\left(\mu_{\alpha}, \sigma_{\alpha}\right)
$$

$$
\mu_{\alpha} \sim \operatorname{Normal}(0,1.5)
$$

$$
\sigma_{\alpha} \sim \text { Exponential(2) }
$$

$$
\beta_{\text {reward,subject }} \sim \operatorname{Normal}\left(\mu_{\text {reward }}, \sigma_{\text {reward }}\right)
$$

$$
\mu_{\text {reward }} \sim \operatorname{Normal}(0,1)
$$

$$
\sigma_{\text {reward }} \sim \text { Exponential }(2)
$$

$$
\beta_{\text {quad_reward,subject }} \sim \operatorname{Normal}\left(\mu_{\text {quad_reward }}, \sigma_{\text {quad_reward }}\right)
$$

$$
\begin{gathered}
\mu_{\text {quad_reward }} \sim \operatorname{Normal}(0,1) \\
\sigma_{\text {quad_reward }} \sim \operatorname{Exponential}(2) \\
\beta_{\text {effort,subject }} \sim \operatorname{Normal}\left(\mu_{\text {effort }}, \sigma_{\text {effort }}\right) \\
\mu_{\text {effort }} \sim \operatorname{Normal}(0,1) \\
\sigma_{\text {effort }} \sim \operatorname{Exponential(}(2)
\end{gathered}
$$

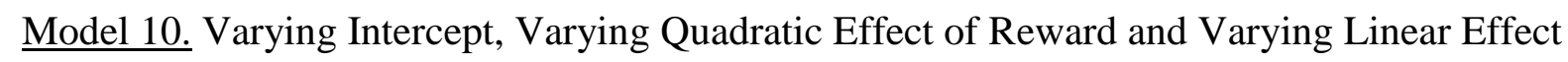
of Effort

Equation $\mathrm{S10}$

$$
Y_{\text {subject,trial }} \sim \operatorname{Bernoulli}\left(p_{\text {subject,trial }}\right)
$$

$$
\begin{aligned}
p_{\text {subject,trial }}= & \operatorname{logistic}\left(\alpha_{\text {subject }}+\beta_{\text {quad_reward,subject }} \text { reward }_{\text {trial }}{ }^{2}\right. \\
& \left.+\beta_{\text {effort,subject }} \text { effort }_{\text {trial }}\right)
\end{aligned}
$$




$$
\begin{gathered}
\alpha_{\text {subject }} \sim \operatorname{Normal}\left(\mu_{\alpha}, \sigma_{\alpha}\right) \\
\mu_{\alpha} \sim \operatorname{Normal}(0,1.5) \\
\sigma_{\alpha} \sim \operatorname{Exponential}(2)
\end{gathered}
$$

$$
\beta_{\text {quad_reward,subject }} \sim \operatorname{Normal}\left(\mu_{\text {quad_reward }}, \sigma_{\text {quad_reward }}\right)
$$

$$
\mu_{\text {quad_reward }} \sim \operatorname{Normal}(0,1)
$$

$$
\sigma_{\text {quad_reward }} \sim \text { Exponential(2) }
$$

$$
\beta_{\text {effort }, \text { subject }} \sim \operatorname{Normal}\left(\mu_{\text {effort }}, \sigma_{\text {effort }}\right)
$$

$$
\begin{gathered}
\mu_{\text {effort }} \sim \operatorname{Normal}(0,1) \\
\sigma_{\text {effort }} \sim \operatorname{Exponential(2)}
\end{gathered}
$$

Model 11. Varying Intercept, Varying Linear Effects of Reward and Effort and Varying Quadratic Effects of Reward and Effort

\section{Equation S11}

$$
\begin{aligned}
& Y_{\text {subject,trial }} \sim \operatorname{Bernoulli}\left(p_{\text {subject,trial }}\right) \\
& p_{\text {subject,trial }}=\operatorname{logistic}\left(\alpha_{\text {subject }}+\beta_{\text {reward,subject }} \text { reward }_{\text {trial }}+\beta_{\text {effort,subject }} \text { effort }\right. \text { trial } \\
& +\beta_{\text {quad_reward,subject }} \text { reward }_{\text {trial }}{ }^{2}+\beta_{\text {quad_effort,subject }} \text { effort } \text { trial }^{2} \text { ) } \\
& \alpha_{\text {subject }} \sim \operatorname{Normal}\left(\mu_{\alpha}, \sigma_{\alpha}\right) \\
& \mu_{\alpha} \sim \operatorname{Normal}(0,1.5) \\
& \sigma_{\alpha} \sim \text { Exponential(2) } \\
& \beta_{\text {reward,subject }} \sim \operatorname{Normal}\left(\mu_{\text {reward }}, \sigma_{\text {reward }}\right) \\
& \mu_{\text {reward }} \sim \operatorname{Normal}(0,1) \\
& \sigma_{\text {reward }} \sim \text { Exponential(2) } \\
& \beta_{\text {effort,subject }} \sim \operatorname{Normal}\left(\mu_{\text {effort }}, \sigma_{\text {effort }}\right) \\
& \mu_{\text {effort }} \sim \operatorname{Normal}(0,1) \\
& \sigma_{\text {effort }} \sim \text { Exponential(2) }
\end{aligned}
$$




$$
\beta_{\text {quad_reward,subject }} \sim \operatorname{Normal}\left(\mu_{\text {quad_reward }}, \sigma_{\text {quad_reward }}\right)
$$

$$
\mu_{\text {quad_reward }} \sim \operatorname{Normal}(0,1)
$$

$$
\sigma_{\text {quad_reward }} \sim \text { Exponential(2) }
$$

$$
\beta_{\text {quad_effort,subject }} \sim \operatorname{Normal}\left(\mu_{\text {quad_effort }}, \sigma_{\text {quad_effort }}\right)
$$

$$
\begin{gathered}
\mu_{\text {quad_effort }} \sim \operatorname{Normal}(0,1) \\
\sigma_{\text {quad_effort }} \sim \operatorname{Exponential(2)}
\end{gathered}
$$

$\underline{\text { Model 12. Varying Intercept, Varying Quadratic Effects of Reward and Effort }}$ Equation S12

$$
Y_{\text {subject,trial }} \sim \operatorname{Bernoulli}\left(p_{\text {subject,trial }}\right)
$$

$$
\begin{aligned}
p_{\text {subject,trial }}= & \operatorname{logistic}\left(\alpha_{\text {subject }}+\beta_{\text {quad_reward,subject }} \text { reward }_{\text {trial }}{ }^{2}\right. \\
& \left.+\beta_{\text {quad_effort,subject }} \text { effort }_{\text {trial }}{ }^{2}\right)
\end{aligned}
$$

$$
\alpha_{\text {subject }} \sim \operatorname{Normal}\left(\mu_{\alpha}, \sigma_{\alpha}\right)
$$

4

$$
\mu_{\alpha} \sim \operatorname{Normal}(0,1.5)
$$$$
\sigma_{\alpha} \sim \text { Exponential(2) }
$$

$$
\beta_{\text {quad_reward,subject }} \sim \operatorname{Normal}\left(\mu_{\text {quad_reward }}, \sigma_{\text {quad_reward }}\right)
$$

$$
\begin{gathered}
\mu_{\text {quad_reward }} \sim \operatorname{Normal}(0,1) \\
\sigma_{\text {quad_reward }} \sim \operatorname{Exponential(2)} \\
\beta_{\text {quad_effort,subject }} \sim \operatorname{Normal}\left(\mu_{\text {effort }}{ }^{2}, \sigma_{\text {effort }^{2}}\right) \\
\mu_{\text {quad_effort }} \sim \operatorname{Normal}(0,1) \\
\sigma_{\text {quad_effort }} \sim \text { Exponential }(2)
\end{gathered}
$$




\section{Prior Predictive Checks}

2 Below are plotted the distributions of all of the parameters used in the eight models, which

3 are expressed and plotted on the logodds scale. Additionally we include plots of the prior

4 predictive distributions for the probability of accepting an offer for an individual subject,

$5 \quad p_{\text {subject }}$. These are on the probability scale. Where the plots show simulated, rather than

6 analytical, distributions, these represent 1000 simulations with 100 hypothetical participants

7 in each. In all cases, the shaded distributions are $66 \%, 95 \%$ and $100 \%$ quantiles.

9 Priors for the intercepts:

10 Shown below are the prior distributions for the two types of intercept parameters, fixed

11 (Figure S1) and varying (Figure S2) intercepts. In both cases we see that the priors chosen

12 represent conservative predictions about the data we would expect to observe. Most

13 importantly, a participant's probability of accepting an offer, $p_{\text {subject }}$, is constrained to be

14 between 0 and 1 . Within that range however the prior density is distributed fairly uniformly,

15 save that it drops off below about 0.2 and above about 0.8 . Overall these priors encode beliefs

16 about participants' average acceptance rates that slightly downweight the likelihood of

17 observing the most extreme values but otherwise are fairly agnostic. 
Figure S1. Prior predictions for the fixed intercept parameter (featured in models 1 and 5), with distribution $\alpha \sim \operatorname{Normal}(0,1.5)$.

(a) shows the analytical distribution of the intercept $\alpha$ itself, whilst (b) shows the implied prior predictions for the probability of accepting an offer for an individual subject.

a

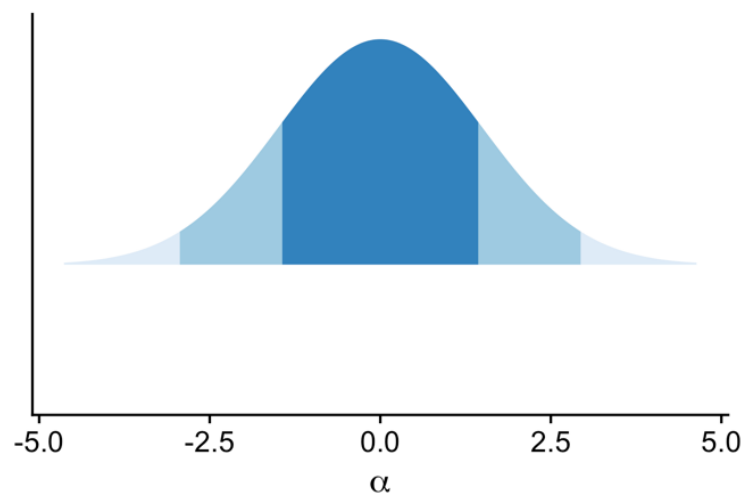

b

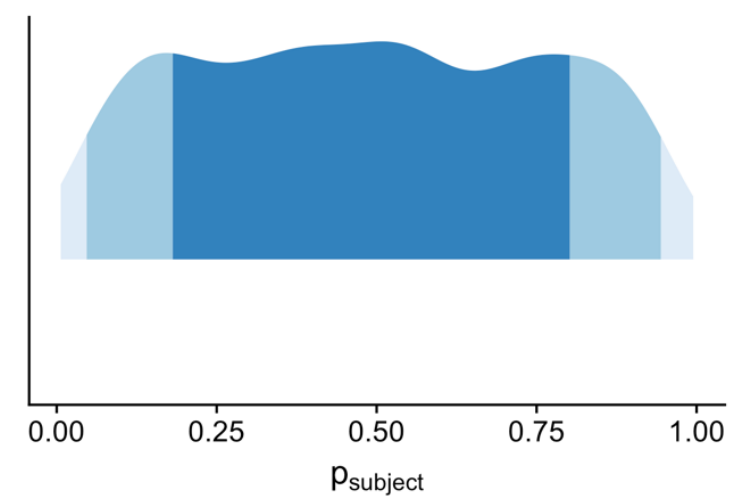

2

Figure S2. Prior predictions for the varying intercept parameters (featured in models 2, 3, 4, 6, 7, 8, 9, 10, 11 and 12), with distributions $\mu_{\alpha} \sim \operatorname{Normal}(0,1.5), \sigma_{\alpha} \sim \operatorname{Exponential(2)}$ and $\alpha_{\text {subject }} \sim \operatorname{Normal}\left(\mu_{\alpha}, \sigma_{\alpha}\right)$. (a) and (b) show the analytical distributions of, respectively, the population level average intercept and the standard deviation of this average; (c) shows the distribution of the (subject level) intercepts themselves; and (d) shows the implied prior predictions for the probability of accepting an offer for an individual subject.

a

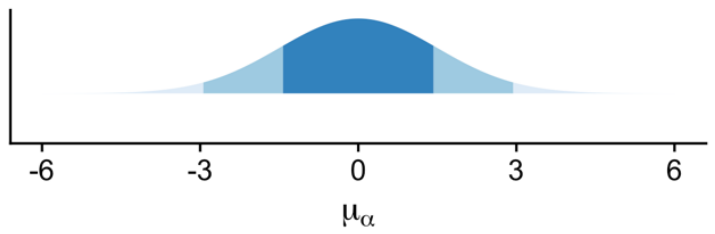

C

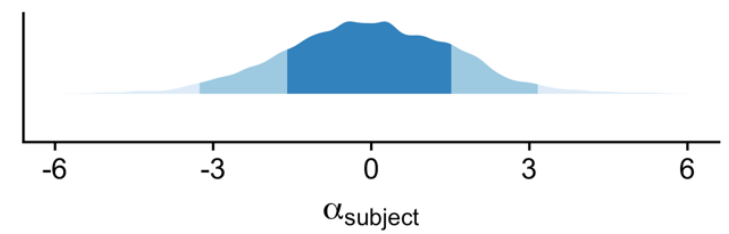

b

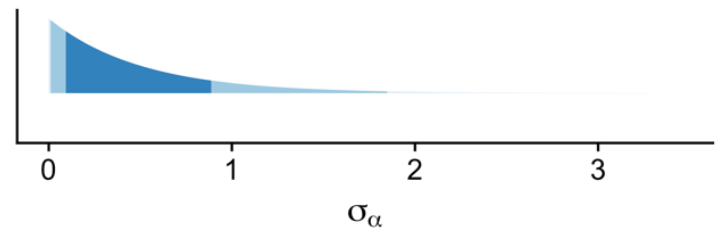

d

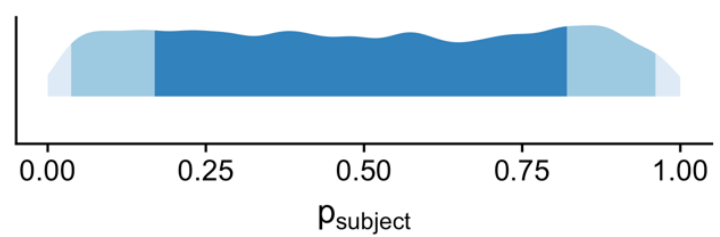

4 
$1 \quad$ Priors for the reward/effort effects

2 Shown below are the prior distributions for the reward/effort sensitivity parameters, for the

3 fixed linear (Figure S3) and varying linear (Figure S4) cases and for the fixed and varying

4 quadratic effort sensitivity parameter (Figure S5).

5

6 The most important plot for interpreting these priors is in the bottom right of each box,

7 labelled b (or d in the case of Figure S4). This shows the prior on the effect of the

8 reward/effort manipulation on the probability scale. Specifically, this is the predicted change

9 in a participant's probability of accepting the offer when one of the manipulations is changed

10 by one level. For example, moving from 3 to 6 points (or 6 to 9 points, etc.), while effort is

11 kept constant, or vice versa moving from $80 \%$ to $60 \%$ effort (or $60 \%$ to $40 \%$ effort, etc.)

12 while reward is kept constant.

13

14 In all cases the priors chosen encode conservative beliefs that the effects, if present, are

15 expected to be approximately in the range $0-0.25$, within which, because of the rightward

16 skew, smaller effects are considered more likely than larger ones. 
Figure S3. Prior predictions for the fixed linear reward and effort sensitivity parameters (featured in models $1,3,5$ and 6), with distribution $\beta \sim \operatorname{Normal}(0,1)$.

(a) shows the analytical distribution of the sensitivity $\beta$ itself, whilst (b) shows the implied predictions for the change in probability of accepting an offer as the reward or effort changes by one level (e.g. from 3 to 6 points, or $80 \%$ to $60 \%$ effort etc.)

a

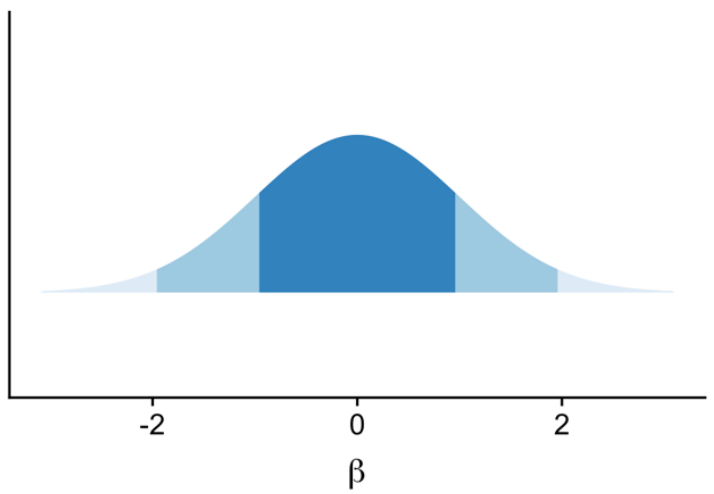

b

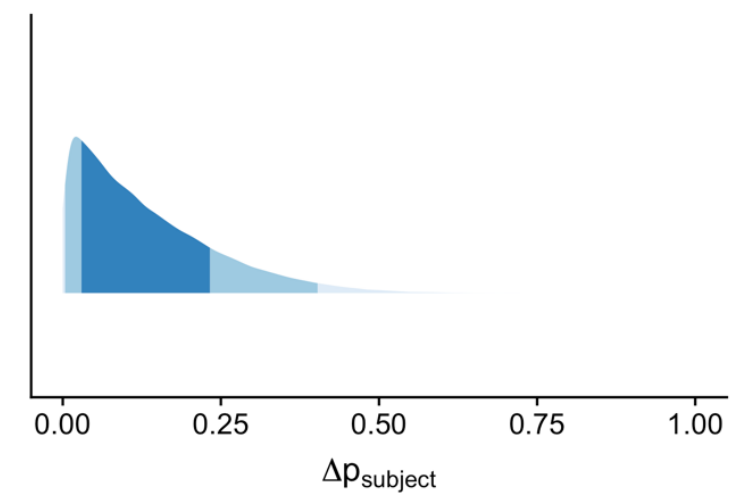

2 
Figure S4. Prior predictions for the varying linear reward and effort sensitivity parameters (featured in models $4,7,8,9,10$ and 11), with distributions $\mu_{\beta} \sim \operatorname{Normal}(0,1)$,

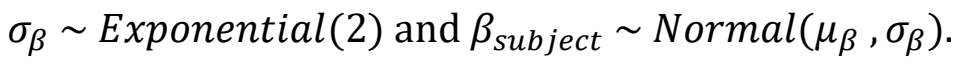

(a) and (b) show the analytical distributions of, respectively, the population level average sensitivity and the standard deviation of this average; (c) shows the distribution of the (subject level) sensitivity parameters themselves; and (d) shows the implied predictions for the change in probability of accepting an offer as the reward or effort changes by one level (e.g. from 3 to 6 points, or $80 \%$ to $60 \%$ effort etc.)

a

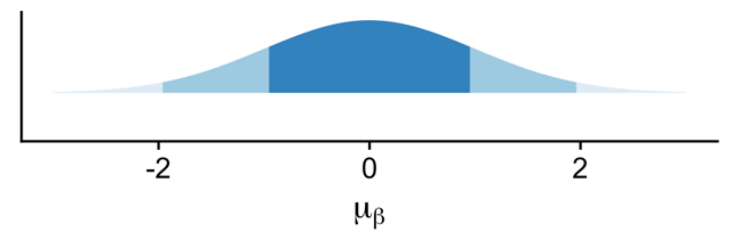

C

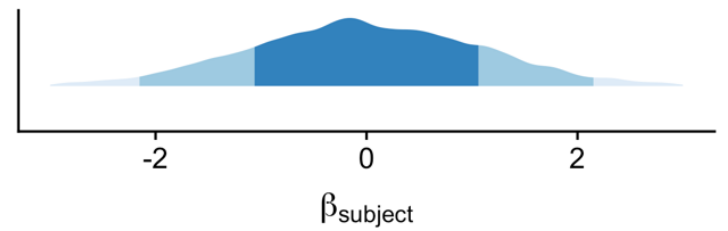

b

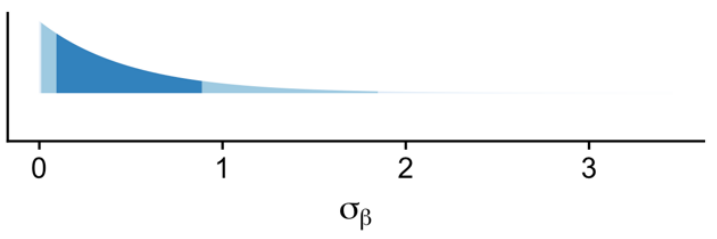

d

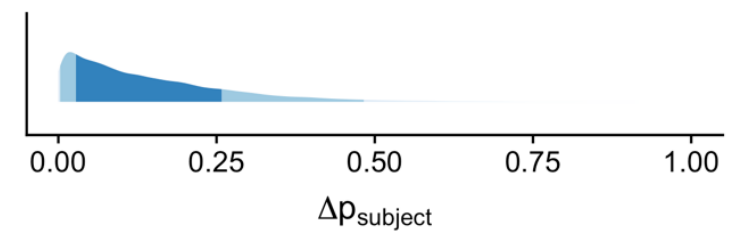

Figure S5. Prior predictions for the quadratic reward/effort sensitivity parameters with distributions $\beta_{\text {quad_effort }} \sim \operatorname{Normal}(0,1)$ (fixed effect in models 5 and 6) and $\mu_{\text {quad_effort }} \sim \operatorname{Normal}(0,1), \sigma_{\text {quad_effort }} \sim \operatorname{Exponential}(2)$ and $\beta_{\text {quad_effort,subject }} \sim \operatorname{Normal}\left(\mu_{\text {quad_effort }}, \sigma_{\text {quad_effort }}\right)$ (varying effects in models 7,8 , $9,10,11$ and 12). The distributions of the sensitivity parameters themselves are the same as for the linear parameters plotted above, in Figure S3(a) for the fixed effect, and in Figure S4(a, b and c) for the varying effects. Below we plot the implied predictions for the change in probability of accepting an offer as the required effort changes by one level (e.g. from $80 \%$ to $60 \%$ effort etc.), for (a) a fixed parameter, and (b) varying

a

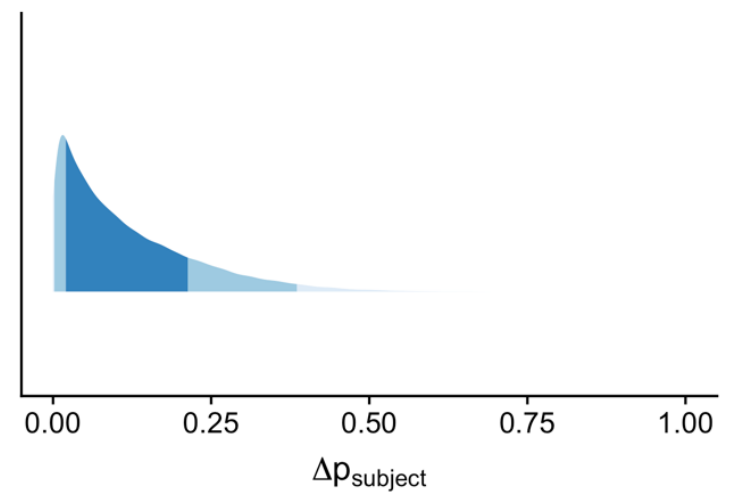

b

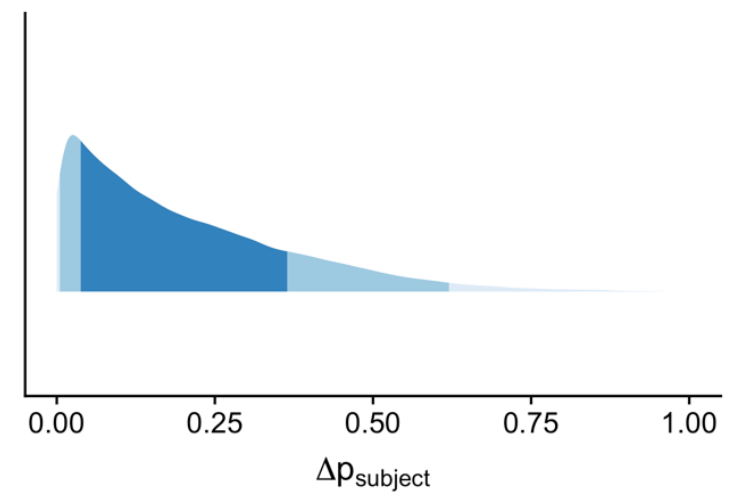




\section{Supplementary Results}

2 Full descriptive statistics for the Cognitive Effort Task

Table S1. Cognitive Effort Task: Proportion of Trials Accepted.

\section{$\mathbf{P}($ accept $)$}

\begin{tabular}{|c|c|c|}
\hline Reward (points) & $\mathbf{N}$ & Mean (SD) \\
\hline 3 & 290 & $0.64(0.37)$ \\
\hline 6 & 290 & $0.84(0.27)$ \\
\hline 9 & 290 & $0.93(0.19)$ \\
\hline 12 & 290 & $0.97(0.12)$ \\
\hline \multicolumn{3}{|l|}{ Effort level } \\
\hline $20 \%$ & 290 & $0.88(0.18)$ \\
\hline $40 \%$ & 290 & $0.86(0.19)$ \\
\hline $60 \%$ & 290 & $0.83(0.21)$ \\
\hline $80 \%$ & 290 & $0.80(0.25)$ \\
\hline \multicolumn{3}{|l|}{ Reward: Effort } \\
\hline $3: 20 \%$ & 290 & $0.72(0.38)$ \\
\hline $3: 40 \%$ & 290 & $0.66(0.39)$ \\
\hline $3: 60 \%$ & 290 & $0.61(0.42)$ \\
\hline $3: 80 \%$ & 290 & $0.58(0.43)$ \\
\hline $6: 20 \%$ & 290 & $0.89(0.25)$ \\
\hline $6: 40 \%$ & 290 & $0.86(0.27)$ \\
\hline
\end{tabular}




\begin{tabular}{|c|c|c|}
\hline $6: 60 \%$ & 290 & $0.83(0.31)$ \\
\hline $6: 80 \%$ & 290 & $0.77(0.35)$ \\
\hline $9: 20 \%$ & 290 & $0.95(0.17)$ \\
\hline $9: 40 \%$ & 290 & $0.94(0.19)$ \\
\hline $9: 60 \%$ & 290 & $0.92(0.22)$ \\
\hline $9: 80 \%$ & 290 & $0.89(0.26)$ \\
\hline $12: 20 \%$ & 290 & $0.97(0.13)$ \\
\hline $12: 40 \%$ & 290 & $0.98(0.11)$ \\
\hline $12: 60 \%$ & 290 & $0.96(0.14)$ \\
\hline $12: 80 \%$ & 290 & $0.95(0.17)$ \\
\hline
\end{tabular}

1

2

3

4

5

6

7

8

9

10

11

12

13

14 


\begin{tabular}{|c|c|c|}
\hline \multicolumn{3}{|c|}{$\mathbf{P}($ success $)$} \\
\hline Reward (points) & $\mathbf{N}$ & Mean (SD) \\
\hline 3 & 273 & $0.86(0.18)$ \\
\hline 6 & 287 & $0.89(0.13)$ \\
\hline 9 & 289 & $0.91(0.10)$ \\
\hline 12 & 289 & $0.92(0.10)$ \\
\hline \multicolumn{3}{|l|}{ Effort level } \\
\hline $20 \%$ & 289 & $0.92(0.10)$ \\
\hline $40 \%$ & 288 & $0.88(0.12)$ \\
\hline $60 \%$ & 289 & $0.88(0.15)$ \\
\hline $80 \%$ & 287 & $0.92(0.12)$ \\
\hline \multicolumn{3}{|l|}{ Reward: Effort } \\
\hline $3: 20 \%$ & 255 & $0.90(0.20)$ \\
\hline $3: 40 \%$ & 247 & $0.84(0.26)$ \\
\hline $3: 60 \%$ & 227 & $0.84(0.25)$ \\
\hline 3: $80 \%$ & 218 & $0.87(0.24)$ \\
\hline $6: 20 \%$ & 280 & $0.91(0.16)$ \\
\hline $6: 40 \%$ & 278 & $0.88(0.19)$ \\
\hline $6: 60 \%$ & 271 & $0.86(0.21)$ \\
\hline $6: 80 \%$ & 263 & $0.93(0.17)$ \\
\hline
\end{tabular}




\begin{tabular}{|c|c|c|}
\hline $9: 20 \%$ & 287 & $0.94(0.13)$ \\
\hline $9: 40 \%$ & 285 & $0.88(0.17)$ \\
\hline $9: 60 \%$ & 281 & $0.89(0.18)$ \\
\hline $9: 80 \%$ & 277 & $0.93(0.14)$ \\
\hline $12: 20 \%$ & 288 & $0.94(0.11)$ \\
\hline $12: 40 \%$ & 288 & $0.90(0.16)$ \\
\hline $12: 60 \%$ & 287 & $0.91(0.17)$ \\
\hline $12: 80 \%$ & 285 & $0.93(0.13)$ \\
\hline
\end{tabular}

1

2

3

4

5

6

7

8

9

10

11

12

13

14

15

16

17 


\begin{tabular}{|c|c|c|}
\hline \multicolumn{3}{|c|}{ Proportional completion time } \\
\hline Reward (points) & $\mathbf{N}$ & Mean (SD) \\
\hline 3 & 273 & $0.84(0.06)$ \\
\hline 6 & 286 & $0.84(0.05)$ \\
\hline 9 & 289 & $0.84(0.05)$ \\
\hline 12 & 289 & $0.83(0.05)$ \\
\hline \multicolumn{3}{|l|}{ Effort level } \\
\hline $20 \%$ & 289 & $0.80(0.07)$ \\
\hline $40 \%$ & 288 & $0.85(0.06)$ \\
\hline $60 \%$ & 288 & $0.86(0.05)$ \\
\hline $80 \%$ & 287 & $0.84(0.05)$ \\
\hline \multicolumn{3}{|l|}{ Reward: Effort } \\
\hline $3: 20 \%$ & 255 & $0.80(0.08)$ \\
\hline $3: 40 \%$ & 247 & $0.85(0.07)$ \\
\hline $3: 60 \%$ & 225 & $0.86(0.06)$ \\
\hline $3: 80 \%$ & 216 & $0.84(0.06)$ \\
\hline $6: 20 \%$ & 280 & $0.80(0.07)$ \\
\hline 6: $40 \%$ & 278 & $0.85(0.06)$ \\
\hline $6: 60 \%$ & 268 & $0.86(0.06)$ \\
\hline
\end{tabular}




\begin{tabular}{|c|c|c|}
\hline $6: 80 \%$ & 263 & $0.85(0.06)$ \\
\hline $9: 20 \%$ & 286 & $0.80(0.07)$ \\
\hline $9: 40 \%$ & 285 & $0.85(0.06)$ \\
\hline $9: 60 \%$ & 278 & $0.86(0.05)$ \\
\hline $9: 80 \%$ & 277 & $0.84(0.05)$ \\
\hline $12: 20 \%$ & 288 & $0.80(0.07)$ \\
\hline $12: 40 \%$ & 288 & $0.84(0.06)$ \\
\hline $12: 60 \%$ & 287 & $0.85(0.06)$ \\
\hline $12: 80 \%$ & 285 & $0.84(0.06)$ \\
\hline
\end{tabular}

3 Bayesian Modelling

4

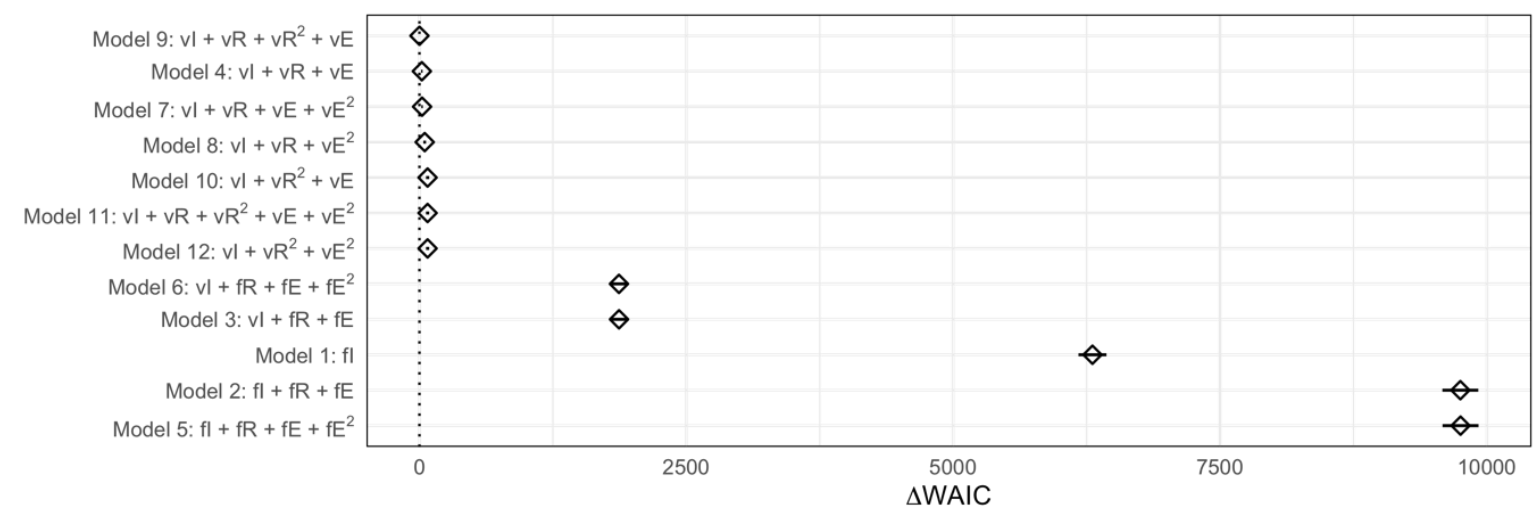

Figure S6. Differences in WAIC relative to the best performing model (Model 4). Full plot of all eight models that were considered. This figure is a counterpart to Figure 6 in the main text.

5 

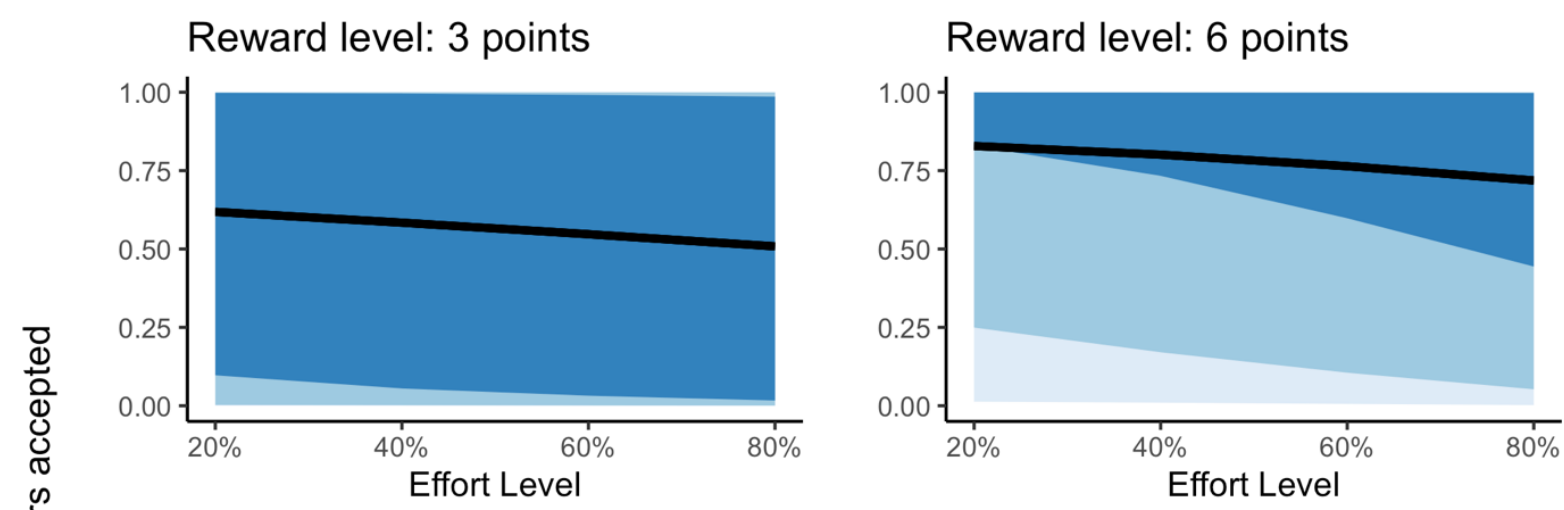

Reward level: 9 points

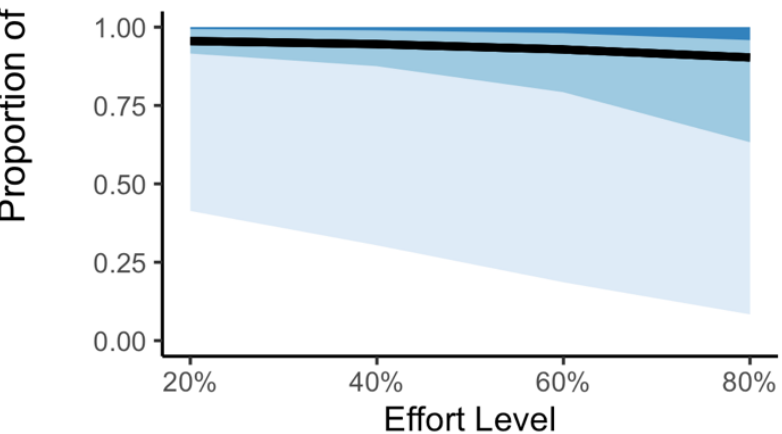

Reward level: 12 points

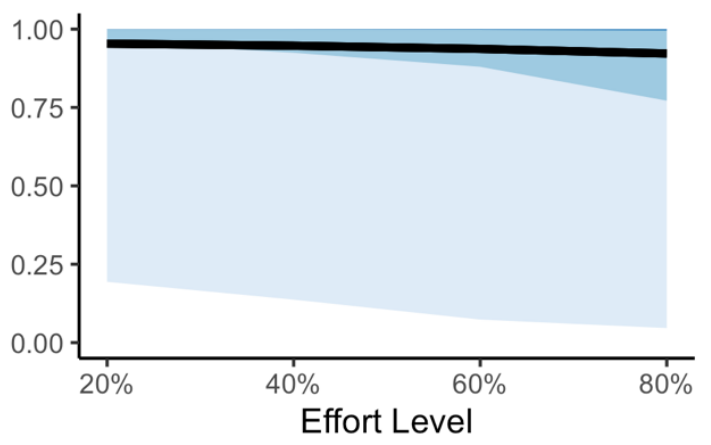

Quantile $95 \%$ $80 \%$ $50 \%$

Figure S7. Posterior predictions for Model $9\left(\mathrm{vI}+\mathrm{vR}+\mathrm{v} R^{2}+\mathrm{vE}\right)$. Plots show mean (black line) and posterior quantiles $(95 \%, 80 \%$ and $50 \%)$ of the predicted probability of accepting an offer across each level of reward and effort. Note that these are predictions for simulated new participants and therefore incorporate uncertainty not just about the average effect of the manipulations in the population, but also about the behaviour of individual participants. 
2 We considered four factor structures:

3

4

10 These are shown graphically in Figure S8

11

12

13 We ran confirmatory factor analyses with each of these models and then compared their

14 relative model fits using three metrics - overall log likelihood, Akaike's Information

15 Criterion and the Bayesian Information Criterion. The results are presented in the Table S4

16 below. The 'Full' structure, with a distinct latent factor for each questionnaire, consistently

17 fitted the data best across all three measures.

18

19

20

21

22

23

24 
Table S4. Results of model comparison for the confirmatory factor analysis.

\begin{tabular}{|l|c|c|c|}
\hline \multicolumn{1}{|c|}{ Factor Structure } & Log Likelihood & AIC & BIC \\
\hline Full structure & -27237 & 54756 & 55273 \\
\hline MH symptoms & -27741 & 55740 & 56213 \\
grouped & & & \\
\hline Cognitive domain & -27827 & 55912 & 56386 \\
\hline 'P'-like structure & -28281 & 56814 & 57277 \\
\hline
\end{tabular}

1

2

3 Using this winning factor structure, we then constructed a structural equation model to

4 predict our three subject-level parameters from the computational model using the latent

5 factors for the questionnaires, plus the demographics measures (age and level of education).

6 The results are presented in Table S5 below. 
a

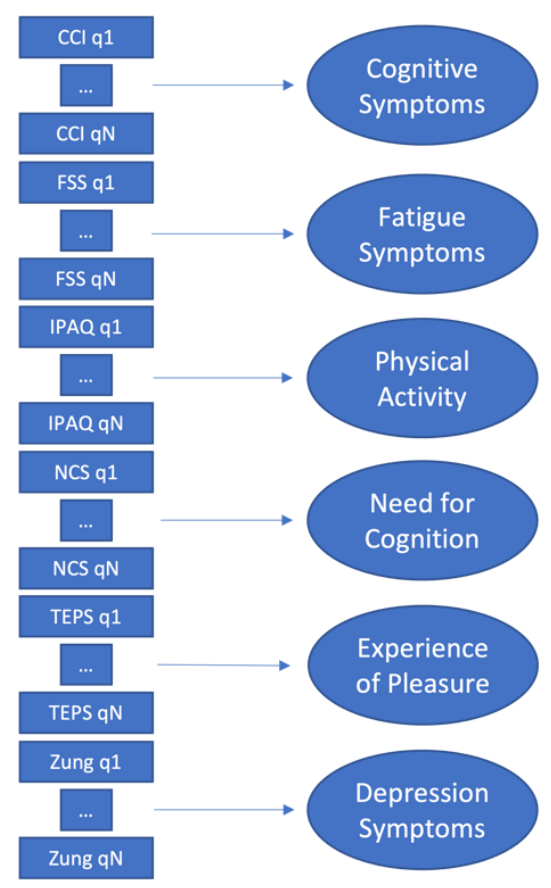

c

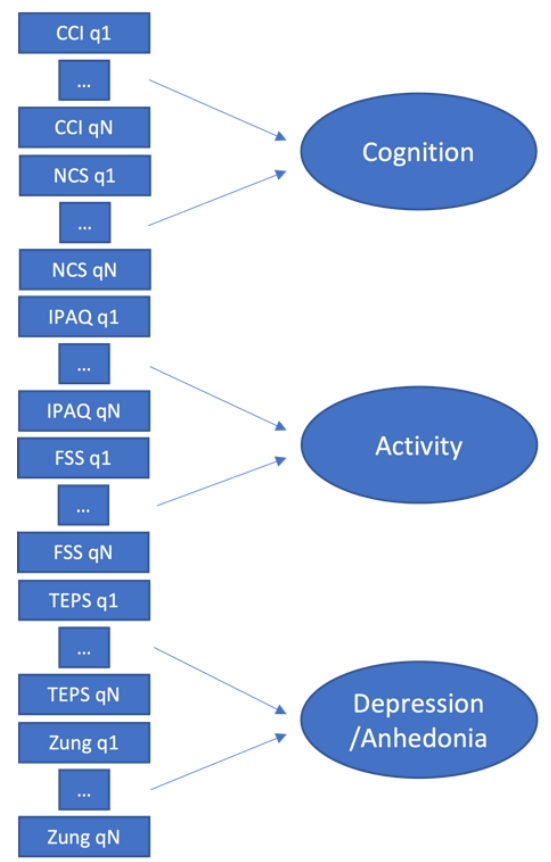

b

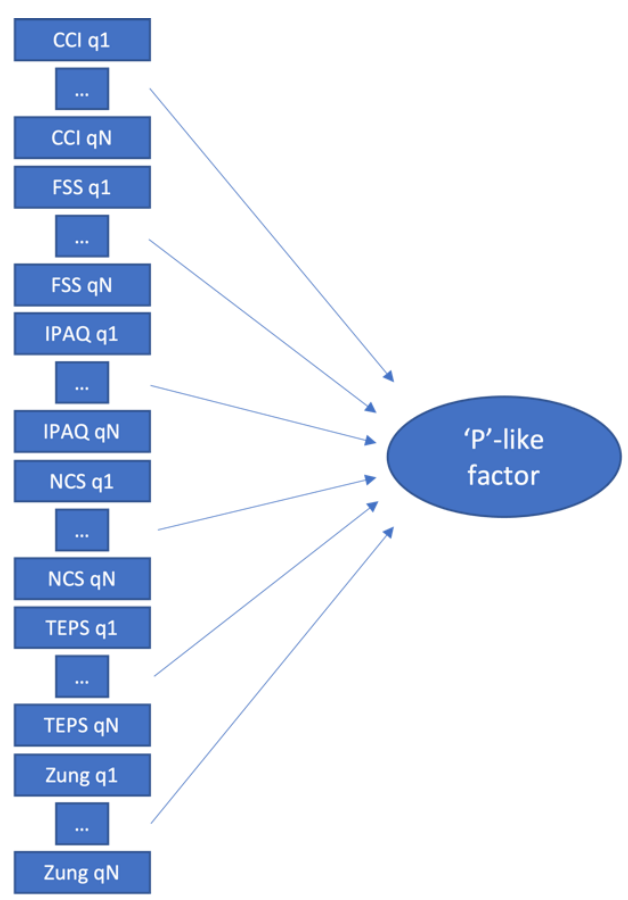

d

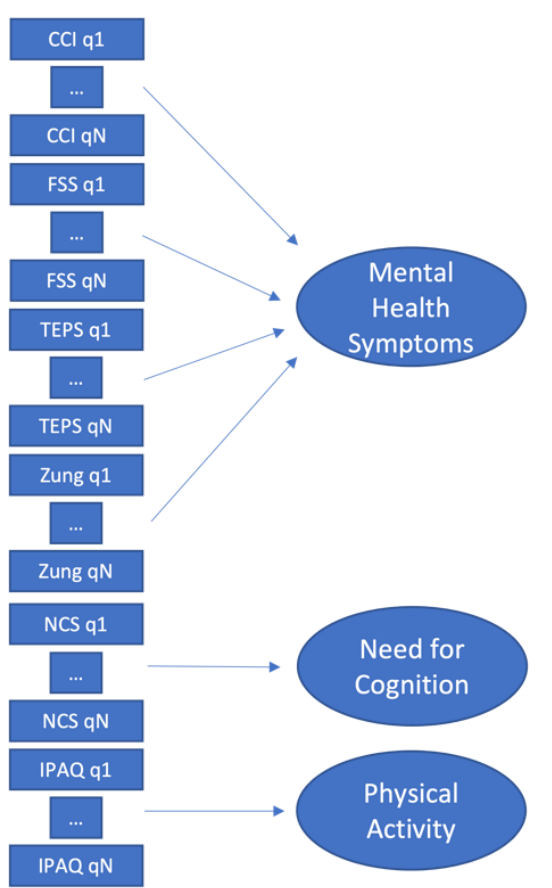

Figure S8. The four factor structures compared in the confirmatory factor analysis. (a) A full factor structure, with a distinct latent factor for each questionnaire. (b) A minimal factor structure, with just a single factor onto which all the questions loaded, corresponding to a 'p'-like factor. (c) An intermediate structure in which questionnaires were grouped by broad cognitive domain. (d) Another intermediate structure, in which the questionnaires directly relevant to mental health symptoms were grouped together. 


\begin{tabular}{|c|c|c|c|}
\hline Path & $\begin{array}{c}\text { Standardised } \\
\text { Coefficient }\end{array}$ & $z$-score & $p$ \\
\hline \multicolumn{4}{|l|}{ Intercept $\rightarrow$} \\
\hline - Age & 0.09 & 0.81 & .42 \\
\hline - $\quad$ Education & -0.219 & 1.68 & .09 \\
\hline - Cognitive Symptoms & -0.006 & 0.02 & .99 \\
\hline - $\quad$ Fatigue Symptoms & -0.388 & 1.31 & .19 \\
\hline - $\quad$ Physical Activity & 0.035 & 0.14 & .99 \\
\hline - $\quad$ Need for Cognition & -0.184 & 0.75 & .46 \\
\hline - Experience of Pleasure & 0.431 & 1.58 & .11 \\
\hline - $\quad$ Depression Symptoms & 0.208 & 0.52 & .61 \\
\hline \multicolumn{4}{|l|}{ Linear reward sensitivity $\rightarrow$} \\
\hline - Age & -0.016 & 0.38 & .71 \\
\hline - Education & 0.091 & 1.84 & .07 \\
\hline - $\quad$ Cognitive Symptoms & 0.162 & 1.14 & .25 \\
\hline - $\quad$ Fatigue Symptoms & -0.077 & 0.69 & .49 \\
\hline - $\quad$ Physical Activity & 0.198 & 1.89 & .06 \\
\hline - $\quad$ Need for Cognition & 0.289 & 3.01 & $.003 * *$ \\
\hline - Experience of Pleasure & -0.192 & 1.83 & .07 \\
\hline - $\quad$ Depression Symptoms & 0.019 & 0.12 & .90 \\
\hline & & & \\
\hline
\end{tabular}




\begin{tabular}{|c|c|c|c|}
\hline Quadratic reward sensitivity $\rightarrow$ & & & \\
\hline - Age & -0.004 & 0.33 & .75 \\
\hline - $\quad$ Education & 0.020 & 1.46 & .15 \\
\hline - Cognitive Symptoms & 0.038 & 0.96 & .34 \\
\hline - $\quad$ Fatigue Symptoms & -0.003 & 0.09 & .93 \\
\hline - $\quad$ Physical Activity & 0.025 & 0.91 & .33 \\
\hline - $\quad$ Need for Cognition & 0.060 & 2.22 & $.03 *$ \\
\hline - Experience of Pleasure & -0.055 & 1.85 & .06 \\
\hline - $\quad$ Depression Symptoms & -0.017 & 0.39 & .70 \\
\hline - Age & -0.008 & 0.43 & .67 \\
\hline - Education & 0.026 & 1.15 & .25 \\
\hline - $\quad$ Cognitive Symptoms & 0.013 & 0.21 & .83 \\
\hline - $\quad$ Fatigue Symptoms & 0.039 & 0.77 & .44 \\
\hline - $\quad$ Physical Activity & 0.015 & 0.36 & .72 \\
\hline - $\quad$ Need for Cognition & -0.080 & 1.87 & .06 \\
\hline - $\quad$ Experience of Pleasure & 0.044 & 0.95 & .34 \\
\hline - $\quad$ Depression Symptoms & 0.046 & 0.66 & .51 \\
\hline
\end{tabular}




\begin{tabular}{|c|c|c|c|}
\hline Latent factors & Covariance & $z$-score & $p$ \\
\hline \multicolumn{4}{|l|}{ Cognitive symptoms } \\
\hline Fatigue Symptoms & 0.498 & 4.76 & $<.001 * * *$ \\
\hline Physical Activity & -0.052 & 0.71 & .48 \\
\hline Need for Cognition & -0.246 & 3.29 & $.001 * *$ \\
\hline Experience of Pleasure & -0.110 & 1.52 & .13 \\
\hline Depression Symptoms & 0.710 & 1.58 & $<.001 * * *$ \\
\hline \multicolumn{4}{|l|}{ Fatigue symptoms } \\
\hline Physical Activity & 0.125 & 1.61 & .11 \\
\hline Need for Cognition & -0.137 & 1.84 & .05 \\
\hline Experience of Pleasure & 0.014 & 1.14 & .83 \\
\hline Depression Symptoms & 0.578 & 5.25 & $<.001 * * *$ \\
\hline \multicolumn{4}{|l|}{ Physical Activity } \\
\hline - $\quad$ Need for Cognition & -0.120 & 1.52 & .13 \\
\hline - $\quad$ Experience of Pleasure & -0.183 & 2.06 & $.04 *$ \\
\hline - $\quad$ Depression Symptoms & 0.065 & 0.90 & .37 \\
\hline \multicolumn{4}{|l|}{ Need for Cognition } \\
\hline - $\quad$ Experience of Pleasure & 0.300 & 3.59 & $<.001 * * *$ \\
\hline - $\quad$ Depression Symptoms & -0.263 & 3.63 & $<.001 * * *$ \\
\hline & & & \\
\hline
\end{tabular}




\begin{tabular}{|c|r|r|r|}
\hline Experience of Pleasure & & & \\
\hline$-\quad$ Depression Symptoms & -0.279 & 3.51 & $<.001 * * *$ \\
\hline
\end{tabular}

1

2 
1 Alternative versions of Figures 3, 4 and 5
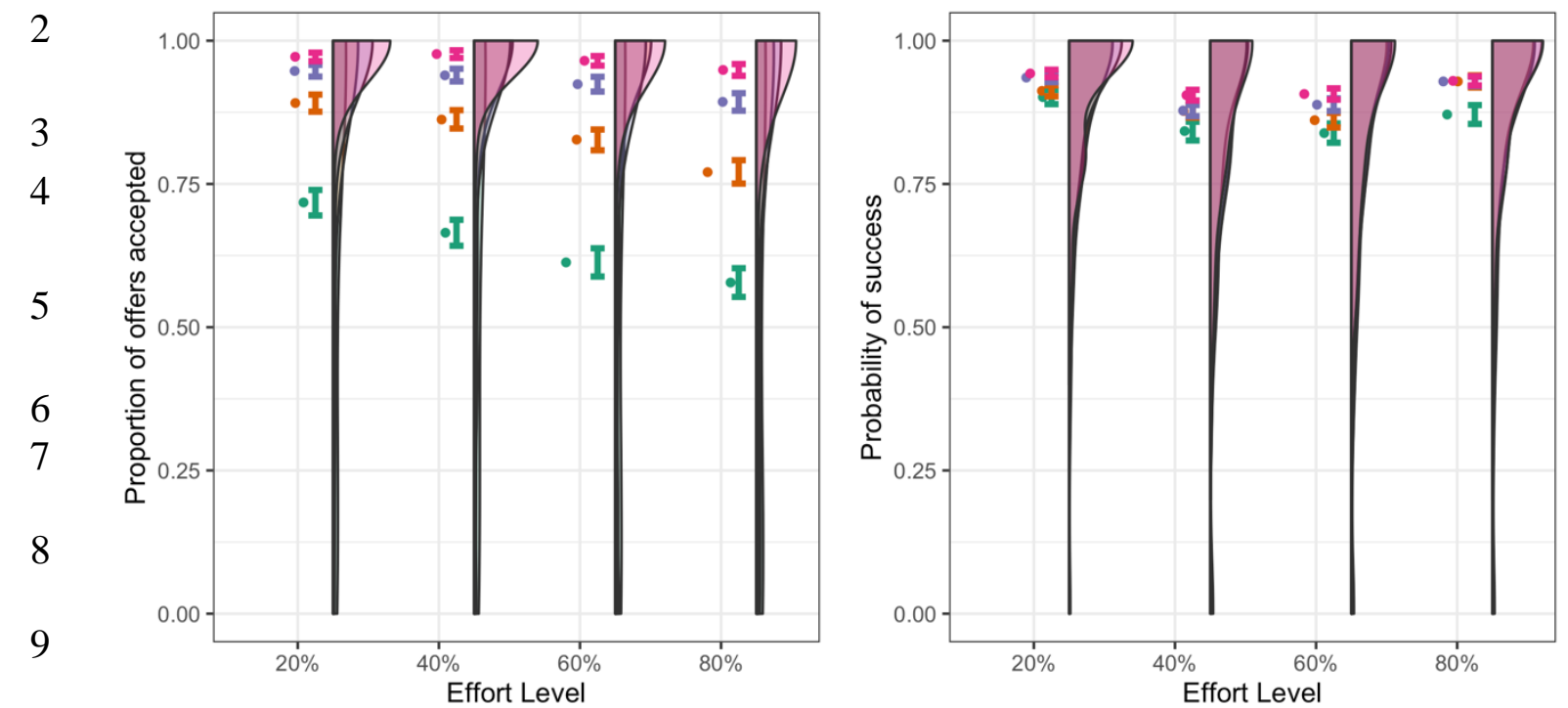

10

11

12

13

14

15

16

17

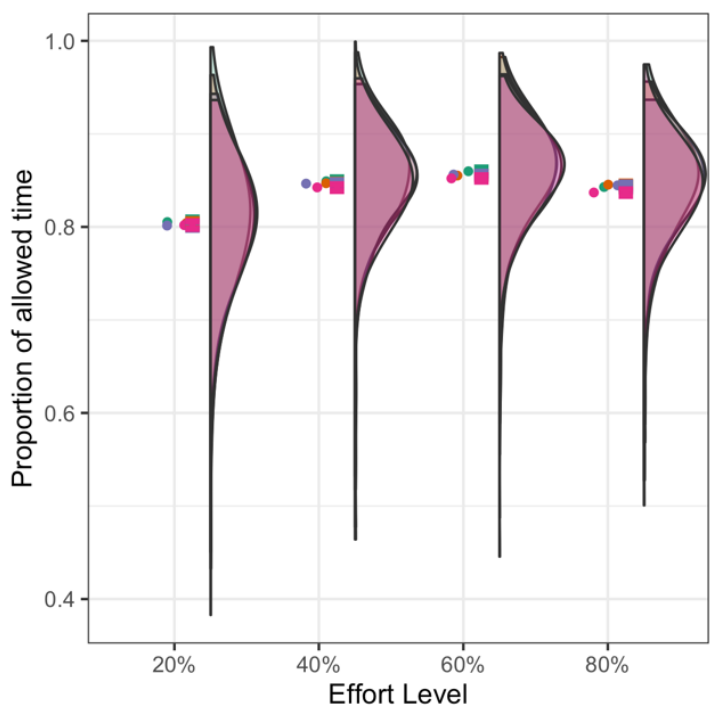

18

Figure S9. Alternative versions of Figures 3, 4 and 5, without faceting. (a) Proportion of offers accepted, (b) Probability of trials completed successfully, and (c) Completion time. 
2 In this section we repeat the modelling analyses using the two runner-up models, named

3 Model $4(v I+v R+v E)$ and Model $7\left(v I+v R+v E+v E^{2}\right)$. First, the posterior parameter

4 estimates are shown below in Figures S10 and S11. We can see that the intercept, reward and

5 effort sensitivity parameter estimates are all substantially similar both across Models 4 and 9

6 and to those in the winning Model $9\left(v \mathrm{I}+\mathrm{vR}+\mathrm{v} R^{2}+\mathrm{vE}\right)$, shown in Figure 8 in the main

7 text. Of note however is that while Models 4 and 7 had similar WAIC scores, Model 7 is less

8 precise than Model 4 in its estimate of the linear effort sensitivity parameter; at the same time

9 the quadratic effort sensitivity parameter both overlaps with zero and is highly colinear with

10 the linear parameter (the correlation between the two is $r=-0.92, p<.001$ ). Together this

11 suggests that the linear and quadratic effort terms are redundant and trade off with one another. Nevertheless, focussing on the linear reward and effort sensitivity parameters we see, as with the winning Model 9, that these do not overlap zero, again according with the earlier ANOVA results).

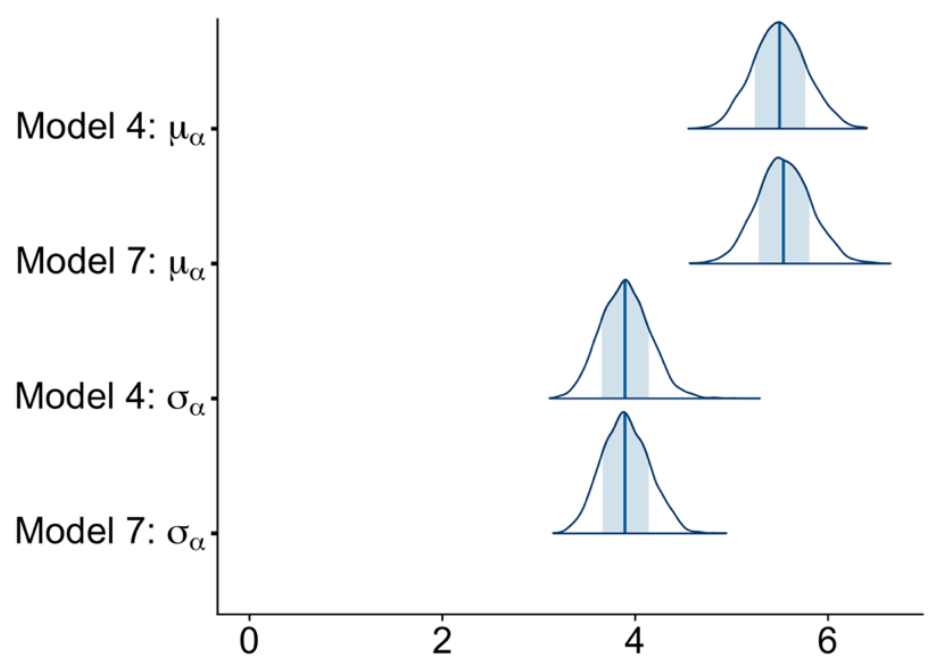

Figure S10. Posterior distributions of the population-level intercept parameters in models 4 $(v I+v R+v E)$ and $7\left(v I+v R+v E+v E^{2}\right)$. The vertical line indicates the mean of each distribution, and the shaded region the $66 \%$ quantile interval. 


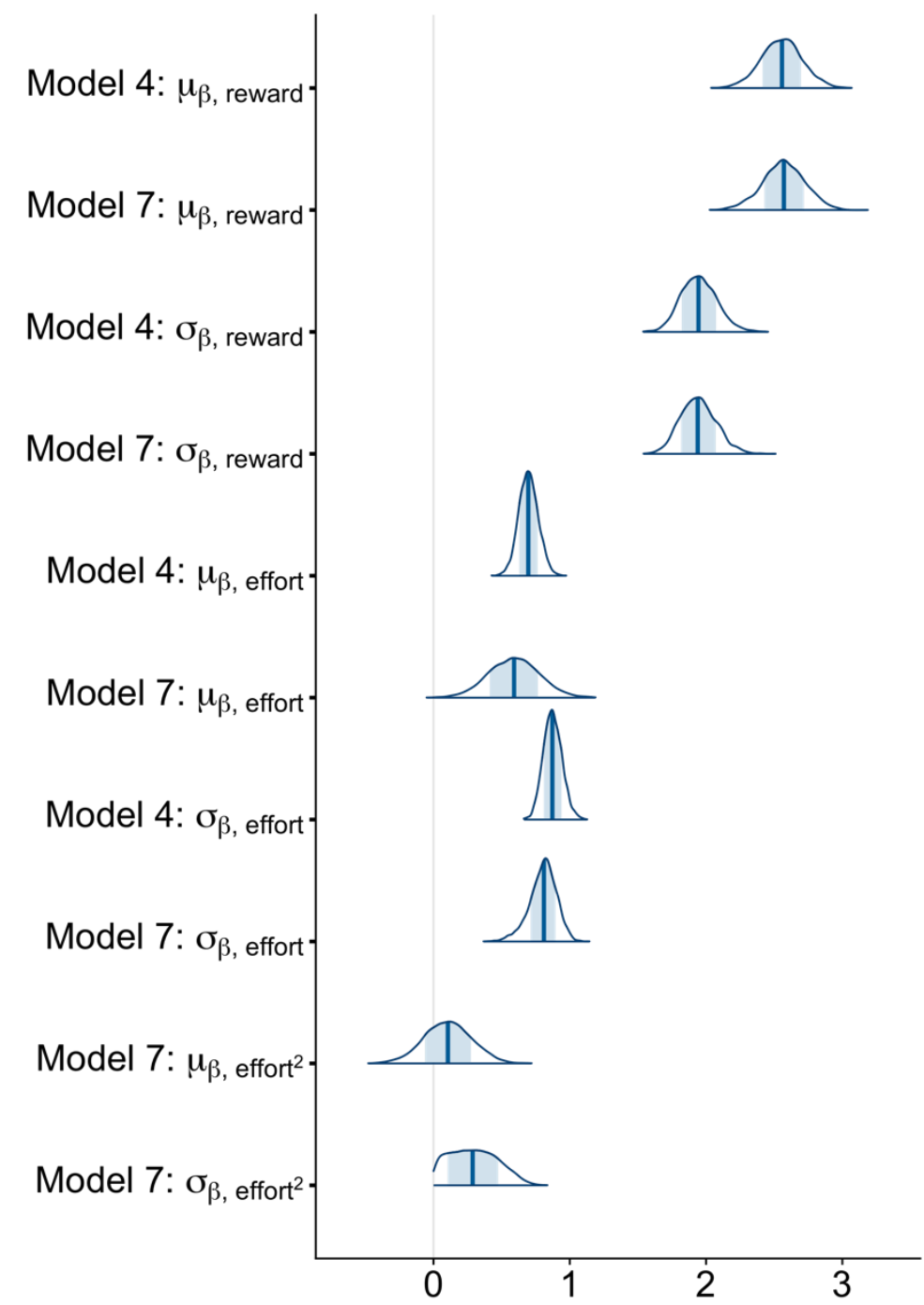

Figure S11. Posterior distributions of the population level reward and effort sensitivity parameters in models $4(v I+v R+v E)$ and $7\left(v I+v R+v E+v E^{2}\right)$. The vertical line indicates the mean of each distribution, and the shaded region the $66 \%$ quantile interval. The quadratic effort sensitivity parameter (Model 7: $\mu_{\beta, \text { effort }}{ }^{2}$ ) substantially overlaps 0 and, additionally, there is a very large negative correlation between this and the model 7 linear effort parameter, $r=-0.92, p<.001$, indicating substantial colinearity. 
1 The posterior predictions from Model $4(v I+v R+v E)$ and Model $7\left(v I+v R+v E+E^{2}\right)$ are

2 shown in Figures S12 and S13. Again we see that both models produce very similar

3 predictions, and both produce a reasonable overall fit to the data while still including

4 substantial uncertainty about the behaviour of an individual participant. In this respect the

5 two models are nearly identical to the winning model considered in the main text, Model 9.

6
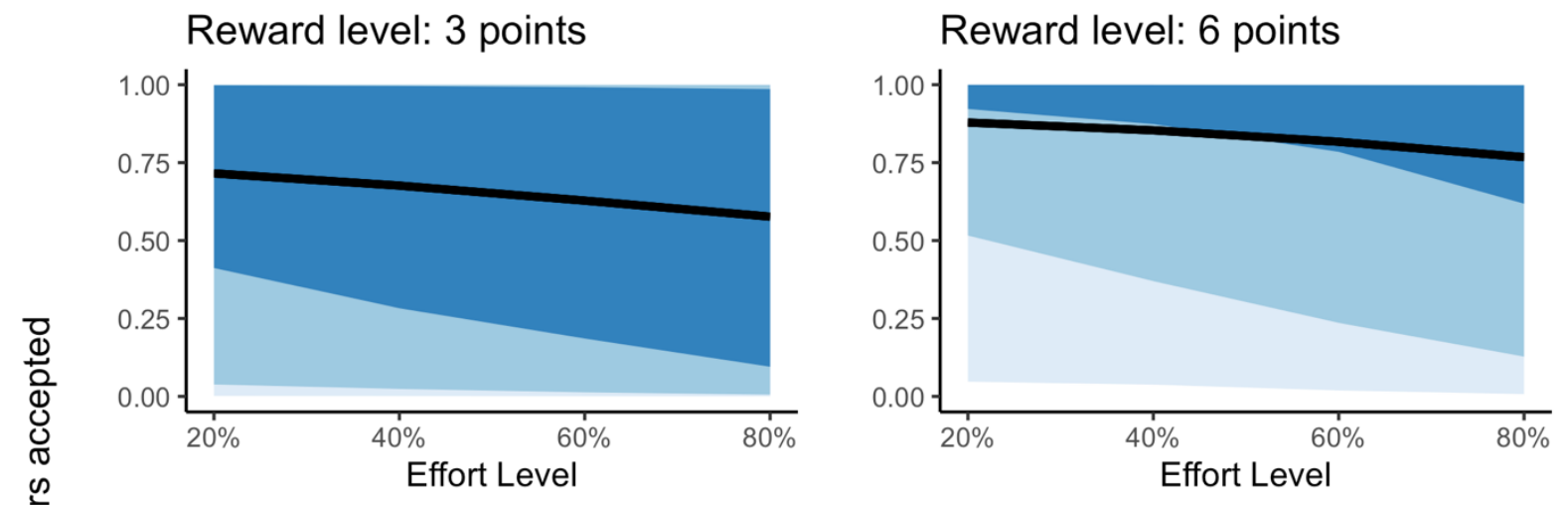

Reward level: 9 points

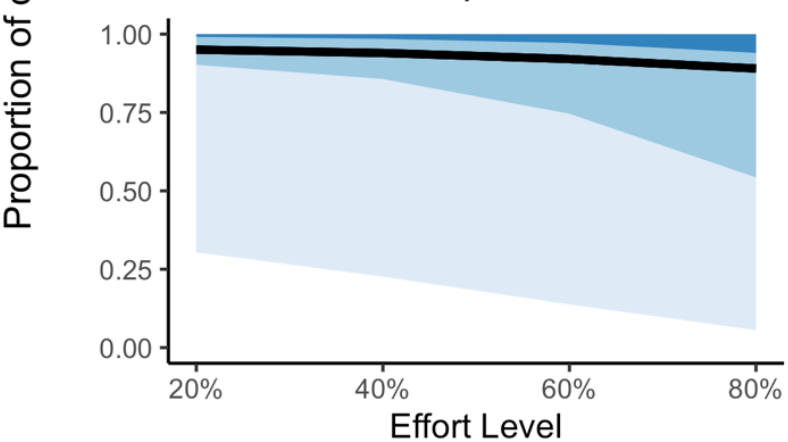

Reward level: 12 points

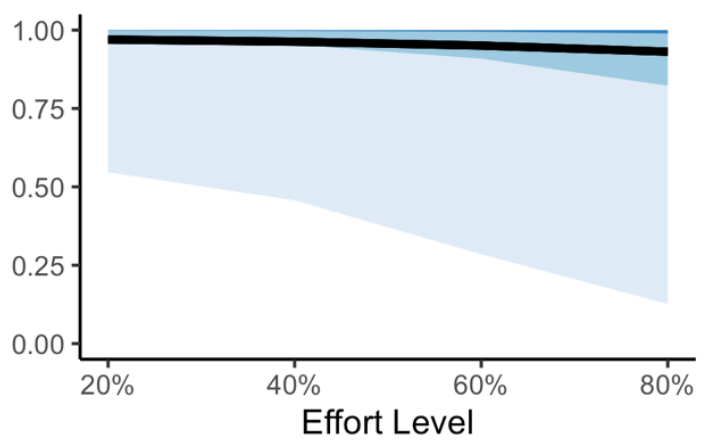

Quantile 95\% $80 \%$ $50 \%$

Figure S12. Posterior predictions from Model $4(v I+v R+v E)$. Plots show mean (black line) and posterior quantiles $(95 \%, 80 \%$ and $50 \%)$ of the predicted probability of accepting an offer across each level of reward and effort. Note that these are predictions for simulated new participants and therefore incorporate uncertainty not just about the average effect of the manipulations in the population, but also about the behaviour of individual participants. 


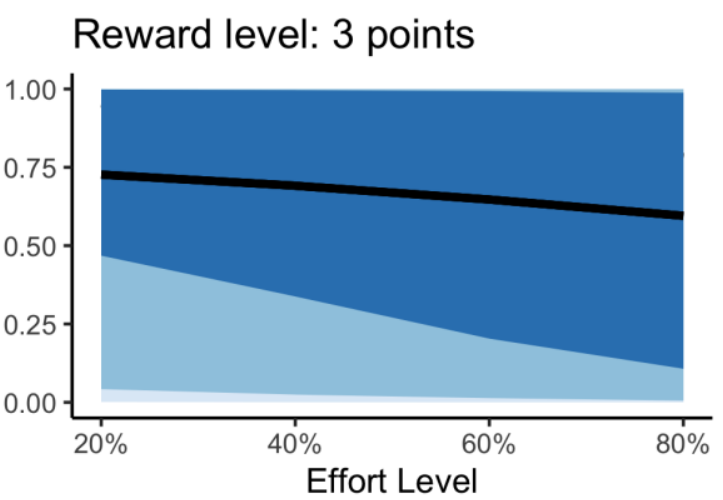

Reward level: 9 points

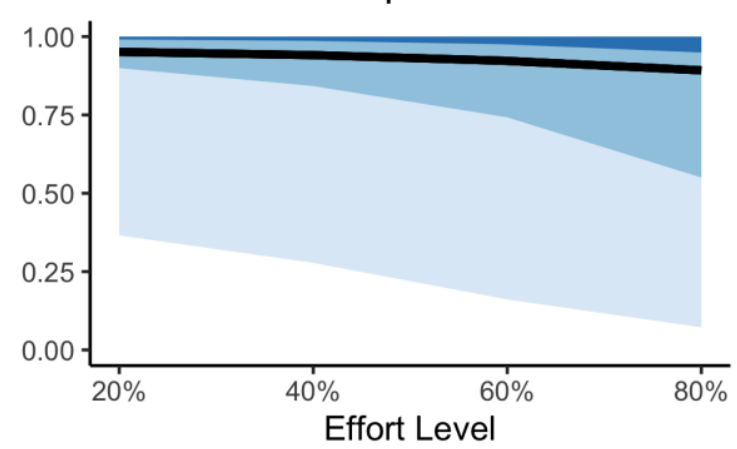

Quantile
Reward level: 6 points

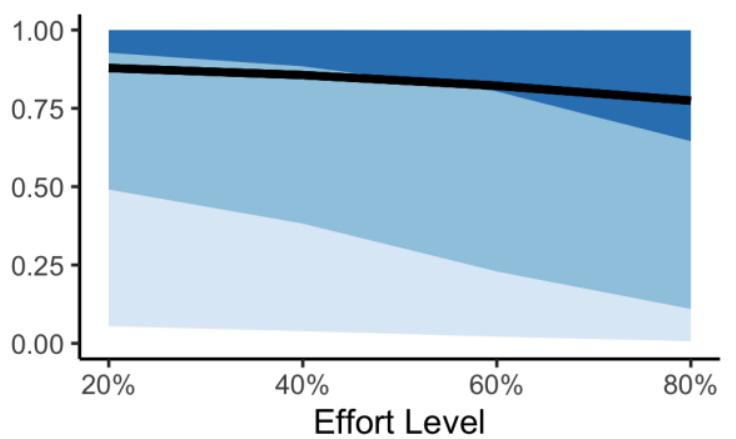

Reward level: 12 points

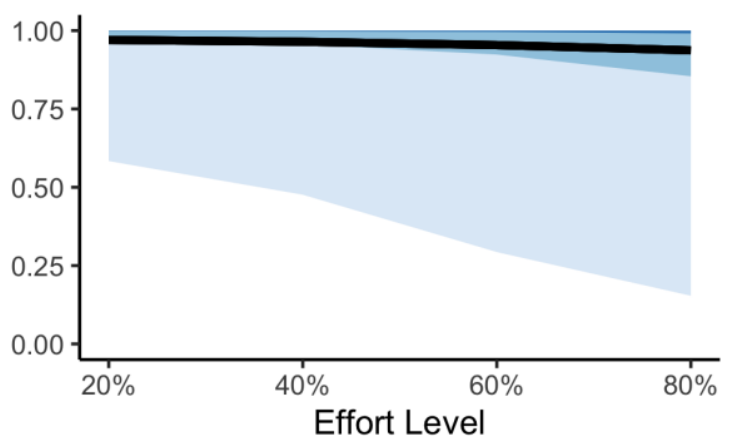

$80 \%$

Figure S13. Posterior predictions from Model $7\left(\mathrm{vI}+\mathrm{vR}+\mathrm{vE}+\mathrm{vE}^{2}\right)$. Plots show mean (black line) and posterior quantiles $(95 \%, 80 \%$ and $50 \%)$ of the predicted probability of accepting an offer across each level of reward and effort. Note that these are predictions for simulated new participants and therefore incorporate uncertainty not just about the average effect of the manipulations in the population, but also about the behaviour of individual participants.

4 Finally, we examined the correlations between the participant-level effort sensitivity

5 parameters (from both Model 4, vI $+v R+v E$, and Model 7, $v I+v R+v E+v E^{2}$ ) and the

6 probability of success on the task (see Figure S12). Again mirroring the results with the

7 winning Model $9\left(v I+v R+v R^{2}+v E\right)$, these correlations were not significantly different

8 from zero $($ Model 4: $r(288)=0.10, p=.09$; Model 7: $r(288)=0.10, p=.07)$, supporting our

9 conclusion that effort sensitivity was not confounded by probability discounting. 
1 Overall these sensitivity analyses support the conclusion that all of the three best performing

2 models on WAIC score lead to very similar inferences, and therefore the results reported in

3 the main text using Model $9\left(v I+v R+v R^{2}+v E\right)$ are robust.

4

5
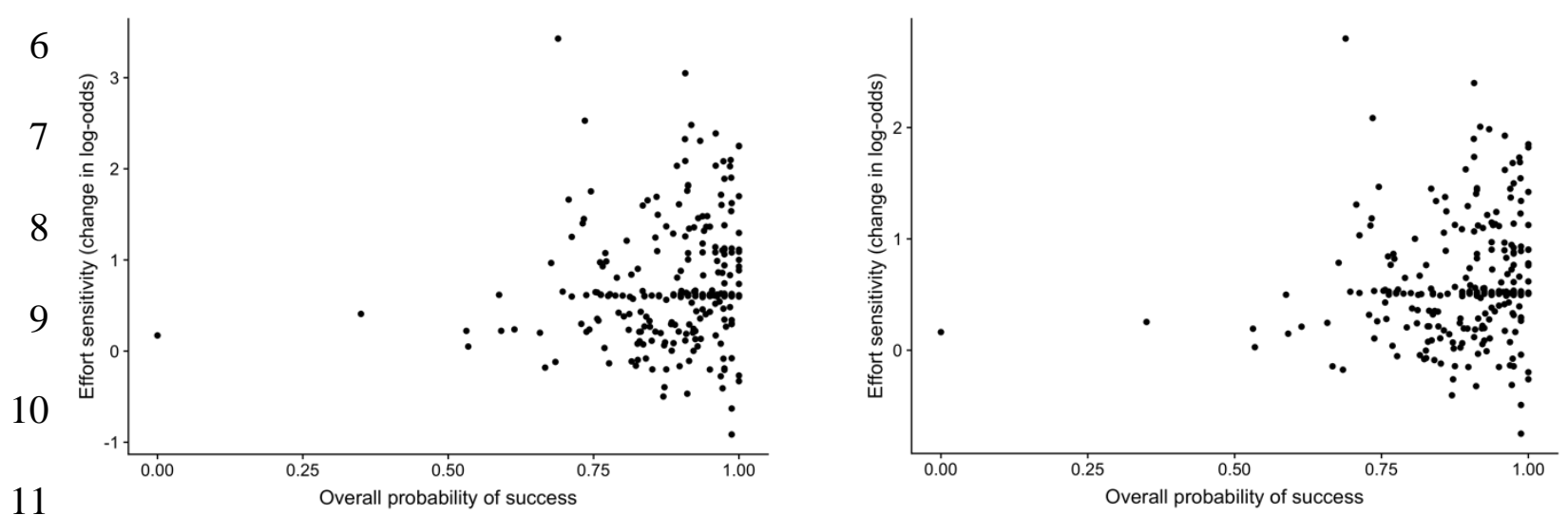

12

Figure S14. Relationship between the probability of success and effort sensitivity. Plots show (left) Model 4 ( $v I+v R+v E)$, and (right) Model $7\left(v I+v R+v E+v^{2}\right)$. The correlation was non-significant, implying that effort sensitivity is not confounded by probability discounting in this task.

17 Further tests of correlations between allowed time, success rate and completion time

Table S7. Cognitive Effort Task: Correlations between time allowed, success rate and completion time for each combination of reward and effort.

\begin{tabular}{|c|c|c|}
\hline \multicolumn{2}{|c|}{ Correlation between time allowed and success rate } \\
\hline Reward: Effort & $r$ & $t$ t-test \\
\hline $3: 20 \%$ & $r=0.07$ & $t(253)=1.15, p=.25$ \\
\hline $3: 40 \%$ & $r=0.14$ & $t(223)=0.86, p=.39$ \\
\hline $3: 60 \%$ & $r=0.06$ & $t(253)=0.72, p=.47$ \\
\hline $3: 80 \%$ & $r=0.05$ & \\
\hline
\end{tabular}




\begin{tabular}{|c|c|c|}
\hline $6: 20 \%$ & $r=0.03$ & $t(278)=0.54, p=.59$ \\
\hline 6: $40 \%$ & $r=0.07$ & $t(276)=1.09, p=.28$ \\
\hline 6: $60 \%$ & $r=0.06$ & $t(266)=0.83, p=.41$ \\
\hline 6: $80 \%$ & $r=0.05$ & $t(261)=0.75, p=.45$ \\
\hline $9: 20 \%$ & $r=0.01$ & $t(284)=0.10, p=.92$ \\
\hline 9: $40 \%$ & $r=0.14$ & $t(283)=2.30, p=.02 *$ \\
\hline 9: $60 \%$ & $r=0.07$ & $t(276)=1.22, p=.22$ \\
\hline $9: 80 \%$ & $r=-.10$ & $t(275)=1.60, p=.11$ \\
\hline $12: 20 \%$ & $r=0.04$ & $t(286)=0.71, p=.48$ \\
\hline $12: 40 \%$ & $r=0.04$ & $t(286)=0.61, p=.54$ \\
\hline $12: 60 \%$ & $r=0.08$ & $t(285)=1.38, p=.17$ \\
\hline $12: 80 \%$ & $r=0.07$ & $t(283)=1.21, p=.23$ \\
\hline
\end{tabular}

\section{Correlation between time allowed and completion time}

\begin{tabular}{|c|c|c|}
\hline Reward: Effort & $\mathbf{r}$ & t-test \\
\hline $3: 20 \%$ & $r=-.40$ & $t(253)=6.95, p<.001 * * *$ \\
\hline $3: 40 \%$ & $r=-.48$ & $t(245)=8.55, p<.001^{* * *}$ \\
\hline $3: 60 \%$ & $r=-.52$ & $t(223)=9.09, p<.001 * * *$ \\
\hline $3: 80 \%$ & $r=-.34$ & $t(214)=5.38, p<.001 * * *$ \\
\hline $6: 20 \%$ & $r=-.42$ & $t(278)=7.69, p<.001 * * *$ \\
\hline $6: 40 \%$ & $r=-.47$ & $t(276)=8.80, p<.001 * * *$ \\
\hline $6: 60 \%$ & $r=-.51$ & $t(266)=9.59, p<.001 * * *$ \\
\hline $6: 80 \%$ & $r=-.51$ & $t(261)=9.69, p<.001 * * *$ \\
\hline $9: 20 \%$ & $r=-.43$ & $t(284)=7.97, p<.001 * * *$ \\
\hline
\end{tabular}




\begin{tabular}{|c|c|c|}
\hline $9: 40 \%$ & $r=-.59$ & $t(283)=12.2, p<.001^{* * *}$ \\
\hline $9: 60 \%$ & $r=-.55$ & $t(276)=10.9, p<.001^{* * *}$ \\
\hline $9: 80 \%$ & $r=-.52$ & $t(275)=10.0, p<.001^{* * *}$ \\
\hline $12: 20 \%$ & $r=-.45$ & $t(286)=8.49, p<.001^{* * *}$ \\
\hline $12: 40 \%$ & $r=-.52$ & $t(286)=10.4, p<.001^{* * *}$ \\
\hline $12: 60 \%$ & $r=-.57$ & $t(285)=11.8, p<.001^{* * *}$ \\
\hline $12: 80 \%$ & $r=-.51$ & $t(283)=10.0, p<.001^{* * *}$ \\
\hline
\end{tabular}

